\title{
REGULARITY PROPERTIES OF COMMUTATORS AND LAYER POTENTIALS ASSOCIATED TO THE HEAT EQUATION
}

\author{
JOHN L. LEWIS AND MARGARET A. M. MURRAY
}

\begin{abstract}
In recent years there has been renewed interest in the solution of parabolic boundary value problems by the method of layer potentials. In this paper we consider graph domains $D=\{(x, t): x>f(t)\}$ in $\mathfrak{R}^{2}$, where the boundary function $f$ is in $I_{1 / 2}(B M O)$. This class of domains would appear to be the minimal smoothness class for the solvability of the Dirichlet problem for the heat equation by the method of layer potentials. We show that, for $1<p<\infty$, the boundary single-layer potential operator for $D$ maps $L^{p}$ into the homogeneous Sobolev space $I_{1 / 2}\left(L^{p}\right)$. This regularity result is obtained by studying the regularity properties of a related family of commutators. Along the way, we prove $L^{p}$ estimates for a class of singular integral operators to which the T1 Theorem of David and Journé does not apply. The necessary estimates are obtained by a variety of real-variable methods.
\end{abstract}

\section{INTRODUCTION}

In recent years, there has been considerable interest in extending the method of layer potentials-which has proved so successful in the solution of elliptic boundary value problems - to the solution of parabolic boundary value problems. Fabes, Riviere, and Brown have established existence, uniqueness, and regularity results for the initial-Dirichlet and initial-Neumann problems in $C^{1}$ and Lipschitz cylinders [FR, $\mathrm{Br} 1, \mathrm{Br} 2]$. These domains have boundaries which are minimally smooth in the spatial variable, but essentially flat with respect to time. Numerous authors have been concerned with the solution of parabolic problems in domains where the time dependence is also somewhat 'rough.' Specifically, Kaufman and $\mathrm{Wu}$ in a series of papers, and Lewis and Silver (see [KW, LeS] for references) have considered domains $D=\{(x, t): x>$ $f(t)\} \subseteq \mathfrak{R}^{2}$, where $f$ is a real-valued continuous function with compact support. Homogeneity considerations would suggest that $f$ must satisfy a halforder smoothness condition in time in order to insure solvability of the Dirichlet problem for the heat equation in $D$. Kaufman-Wu showed that $f \in \operatorname{Lip}_{1 / 2}$

Received by the editors May 18, 1989 and, in revised form, October 20,1989. Presented to the Society at the 853rd meeting in Los Angeles, California, November 18, 1989.

1980 Mathematics Subject Classification (1985 Revision). Primary 42A50, 35K05, 35B65.

The first author was supported in part by the NSF, and by the Commonwealth of Kentucky through the Kentucky EPSCoR program.

The second author was supported in part by the NSF, with travel assistance provided by the Commonwealth of Kentucky through the Kentucky EPSCoR program. 
is not a sufficient condition for the absolute continuity of caloric measure with respect to Lebesgue measure. Lewis-Silver obtained a somewhat stronger Dinitype condition on $f$, which ensures both the absolute continuity of caloric measure and the solvability of the $L^{p}$-Dirichlet problem for the heat equation in terms of a double-layer heat potential when $1<p<\infty$. In particular, the Dini condition on $f$ ensures the $L^{p}$ boundedness of certain commutators with fractional differentiation which are closely related to the double-layer potential and, as a corollary, the $L^{p}$ boundedness of the double-layer potential itself. Because there would appear to be no readily available Rellich-type formula for $\partial D$, the inversion of boundary potentials is carried out using Neumann series; thus much of the hard work of the proof goes into the $L^{p}$ estimates for the commutators.

By virtue of the work of Murray on the $L^{2}$ boundedness of fractional differentiation commutators [Mu1, Mu2], the work of Lewis-Silver may be extended to a class of domains of intermediate smoothness. Specifically, $f \in I_{1 / 2}(\mathrm{BMO})$ (the image of BMO under the half-order Riesz potential) is a necessary and sufficient condition for the $L^{2}$ boundedness of the commutators associated to the double-layer heat potential [Mu1]. The condition $f \in I_{1 / 2}(\mathrm{BMO})$ implies $f \in \operatorname{Lip}_{1 / 2}$, and is implied by the Dini condition of Lewis-Silver. It seems likely that the class of $I_{1 / 2}(\mathrm{BMO})$ domains in $\mathfrak{R}^{2}$ is the minimal smoothness class for solvability of the Dirichlet problem in $L^{2}$ by the method of layer potentials; although in general, inversion is still a problem.

In this paper, we consider the regularity properties of the single-layer heat potential operator in $I_{1 / 2}(\mathrm{BMO})$ domains by studying the regularity properties of a related class of fractional differentiation commutators. A summary of our results is as follows. Recall that the fundamental solution for the heat equation in $\mathfrak{R}^{2}$ is given by

$$
W(x, t)=(4 \pi t)^{-1 / 2} \exp \left(-x^{2} / 4 t\right) \chi_{(0, \infty)}(t) .
$$

Let $f$ be a compactly supported function in $I_{1 / 2}(\mathrm{BMO})$ and let $F \in L^{p}(\mathfrak{R})$ for some $p \in(1, \infty)$. The single-layer potential of $F$ for the interior of $D$ is defined, for $x>f(t)$ and $t \in \mathfrak{R}$, by

$$
S^{i} F(x, t)=\int_{-\infty}^{t} W(x-f(s), t-s) F(s) d s .
$$

The boundary single-layer potential of $F$ is defined for $t \in \mathfrak{R}$ by

$$
S^{b} F(t)=\int_{-\infty}^{t} W(f(t)-f(s), t-s) F(s) d s .
$$

We want to show that $S^{b}$ maps $L^{p}(\Re)$ boundedly into $I_{1 / 2}\left(L^{p}(\Re)\right)$, the homogeneous Sobolev space given by the image of $L^{p}$ under the half-order Riesz potential $I_{1 / 2}$. To do this, we begin by studying a closely related family of commutators. 
For $\alpha \in(0,1)$ define the operator $K_{\alpha}$ by

$$
K_{\alpha} g(t)=\text { p.v. } \int_{-\infty}^{\infty} \frac{(f(s)-f(t))^{2}}{|s-t|^{1+\alpha}} g(s) d s ;
$$

$K_{\alpha}$ is, formally, the commutator $\left[f,\left[f, I_{-\alpha}\right]\right]$. The results in [Mu2] show that if $1<p<\infty$ and $f \in I_{\alpha / 2}(\mathrm{BMO})$, then $K_{\alpha}$ is bounded on $L^{p}(\Re)$. For $1<p<\infty$, we show that under the stronger hypothesis that $f \in I_{\alpha}(\mathrm{BMO}), K_{\alpha}$ actually maps $L^{p}(\Re)$ into $L^{p}(\Re) \cap I_{\alpha}\left(L^{p}(\Re)\right)$. The proof proceeds via a series of steps. First, we show that for $p \geq 2, K_{\alpha}$ maps $L^{p}$ into the homogeneous Besov space $\dot{B}_{p p}^{\alpha}$. For $p=2, \dot{B}_{p p}^{\alpha}=I_{\alpha}\left(L^{2}\right)$, so that we obtain the desired result for $p=2$. Next, we show that the operator $D^{\alpha} K_{\alpha}$ is bounded on $L^{p}, 1<p<\infty$, where $D^{\alpha}$ is a formal inverse for $I_{\alpha}$. The operator $D^{\alpha} K_{\alpha}$ does not satisfy the hypotheses of the T1 Theorem of David and Journe [DJ]; the proof of $L^{p}$ boundedness relies upon the $L^{2}$ result together with the commutator results of Murray [Mu1, Mu2] and a variety of real-variable methods. We then deduce that $K_{\alpha}$ maps $L^{p}$ into $L^{p} \cap I_{\alpha}\left(L^{p}\right)$, and indicate how the proof may be extended to show that $S^{b}$ maps $L^{p}$ into $I_{\alpha}\left(L^{p}\right)$.

The authors wish to thank Russell Brown, Eugene Fabes, and Gregory Verchota for helpful discussions on the subject matter of this paper-but more importantly, for their role in introducing each of us to the other's work on this and related topics.

\section{NOTATION AND PRELIMINARIES}

For functions $\phi$ in the Schwartz class $\mathscr{S}=\mathscr{S}(\mathfrak{R})$ we define the Fourier transform according to the normalization

$$
\widehat{\phi}(\xi)=\int_{\mathfrak{R}} e^{-i x \xi} \phi(x) d x
$$

and we define the Riesz potential operator of order $\alpha \in(0,1)$ by setting

$$
\left(I_{\alpha} \phi\right)^{\wedge}(\xi)=|\xi|^{-\alpha} \widehat{\phi}(\xi)
$$

Properties of the Riesz potentials are discussed in detail in [BL, Chapter 6; St, Chapter 5]. The space BMO of functions of bounded mean oscillation is the Banach space of functions (modulo constants) satisfying

$$
\|b\|_{*}=\sup _{I}|I|^{-1} \int_{I}\left|b(x)-m_{I}(b)\right| d x<\infty, \quad m_{I}(b)=|I|^{-1} \int_{I} b,
$$

where the supremum in (2.3) is taken over all intervals $I$.

The space $I_{\alpha}(\mathrm{BMO})$ is the image of BMO under the Riesz potential $I_{\alpha}$; characterizations of this BMO Sobolev space may be found in [Str] (see also [Mul]). In particular, it is not difficult to see that $I_{\alpha}(\mathrm{BMO})$ is a space of functions (modulo constants), properly contained in the space of Lipschitz functions of order $\alpha$. 
If $f=I_{\alpha}(b)$, with $b \in \mathrm{BMO}$, then $\|f\|_{\operatorname{Lip}_{\alpha}} \leq c_{\alpha}\|b\|_{*}$, where $c_{\alpha}$ depends only upon $\alpha$, and

$$
\|f\|_{\operatorname{Lip}_{\alpha}}=\sup _{x \neq y} \frac{|f(x)-f(y)|}{|x-y|^{\alpha}} .
$$

Moreover, the measure

$$
d \mu(x, t)=\frac{|f(x+t)-f(x)|^{2}}{|t|^{1+2 \alpha}} d x d t
$$

is a Carleson measure on $\mathfrak{R}_{+}^{2}=\{(x, t): x \in \mathfrak{R}, t>0\}$, with norm $\leq c_{\alpha}\|b\|_{*}^{2}$ (cf. [Str, Theorem 3.3]). We note also the proper inclusion $\operatorname{Lip}_{\beta} \subseteq I_{\alpha}(\mathrm{BMO})$ for $1>\beta>\alpha>0$ (see [Str, Theorem 3.4] together with [St, Proposition 10, p. 153]).

The homogeneous Besov spaces may be given numerous equivalent characterizations (see [BL, St]). For our purposes we define, for $\alpha \in(0,1)$ and $p \in(1, \infty)$, the homogeneous Besov space $\dot{B}_{p p}^{\alpha}$ to be the space of distributions $\phi \in \mathscr{S}^{\prime}$ (modulo constants) satisfying

$$
\|\phi\|_{\dot{B}_{p p}^{\alpha}}=\left(\int_{\mathfrak{R}} \int_{0}^{\infty}|\phi(t+h)-\phi(t)|^{p} h^{-1-p \alpha} d h d t\right)^{1 / p}<\infty .
$$

For $p>2$, we have the proper inclusion $I_{\alpha}\left(L^{p}\right) \subseteq \dot{B}_{p p}^{\alpha}$, while for $p<2$ the proper inclusion is reversed: $\dot{B}_{p p}^{\alpha} \subseteq I_{\alpha}\left(L^{p}\right)$. For $p=2, I_{\alpha}\left(L^{2}\right)=\dot{B}_{22}^{\alpha}$. The (nonhomogeneous) Besov space $B_{p p}^{\alpha}$ is simply $\dot{B}_{p p}^{\alpha} \cap L^{p}$; the norm on $B_{p p}^{\alpha}$ is just the sum of the $\dot{B}_{p p}^{\alpha}$ and $L^{p}$ norms.

The following result is crucial to all of our work:

Commutator Theorem. Suppose that $\alpha \in(0,1), f=I_{\alpha}(b), b \in \mathrm{BMO}$. Let $k$ be a positive integer, and let $C_{k}$ denote the operator

$$
C_{k} g(t)=\lim _{\varepsilon \rightarrow 0+} C_{k, \varepsilon} g(t)=\lim _{\varepsilon \rightarrow 0+} \int_{|s-t|>\varepsilon} \frac{(f(s)-f(t))^{k}}{|s-t|^{1+k \alpha}} g(s) d s
$$

with associated maximal operator

$$
C_{k}^{*} g(t)=\sup _{\varepsilon>0}\left|C_{k, \varepsilon} g(t)\right| .
$$

Then $C_{k}, C_{k}^{*}$ are bounded on $L^{p}(\mathfrak{R})$ for $1<p<\infty$, and

$$
\left\|C_{k} g\right\|_{p},\left\|C_{k}^{*} g\right\|_{p} \leq c_{\alpha, p}\|b\|_{*}\|f\|_{\operatorname{Lip}_{\alpha}}^{k-1}\|g\|_{p}
$$

where $c_{\alpha, p}$ depends upon $\alpha, p$ but not upon $k, g, f$.

The estimate (2.8) for $C_{k}$ was obtained in [Mu1] for $k=1, p=2$, and in [Mu2] for $k \geq 2, p=2$. These $L^{2}$ estimates may also be obtained by a 
relatively straightforward application of the $\mathrm{T} 1$ Theorem of David and Journé [DJ]. Furthermore, it is easily seen that, for all $k$, the kernel

$$
A_{k}(s, t)=\frac{(f(s)-f(t))^{k}}{|s-t|^{1+k \alpha}}
$$

of $C_{k}$ satisfies the standard estimates

$$
\begin{gathered}
\left|A_{k}(s, t)\right| \leq\|f\|_{\operatorname{Lip}_{\alpha}|s-t|^{-1}}^{k} \\
\left|A_{k}(s, t)-A_{k}\left(s, t_{0}\right)\right| \leq k c_{\alpha}|| f \|_{\operatorname{Lip}_{a}}^{k}\left|t-t_{0}\right|^{\alpha}\left|s-t_{0}\right|^{-1-\alpha} \\
\text { for } 2\left|t-t_{0}\right| \leq\left|s-t_{0}\right| .
\end{gathered}
$$

From these estimates, together with the $L^{2}$ result for $C_{k}$, it is easy to obtain, first, the $L^{2}$ estimate for $C_{k}^{*}$, and then the weak-type $(1,1)$ estimates for $C_{k}$ and $C_{k}^{*}$ (see [T, Chapter 11] or [St, Chapter 2]).

The $L^{p}$ estimates for $C_{k}$ and $C_{k}^{*}$ now follow by interpolation; to obtain constants independent of $k$, observe that $A_{2}$ is nonnegative and that $\left|A_{k}(s, t)\right| \leq\|f\|_{\text {Lip }_{\alpha}}^{k-2} A_{2}(s, t)$ for $k \geq 2$. We remark that all of these results continue to hold when, for $k \geq 1$, we replace $A_{k}(s, t)$ by $\operatorname{sgn}(s-t) A_{k}(s, t)$.

We conclude this section with a general remark on notation. We use the lowercase letter ' $c$ ' to denote a constant, not necessarily the same at each occurrence; when $c$ depends explicitly on a particular quantity, the dependence will be indicated by a subscript (e.g., $c_{\alpha}$ in (2.12) depends only upon $\alpha$ ). If the dependence of the constant is absolutely clear from the context, the subscript will be deleted.

\section{BESOV SPACE RESUlTS}

Throughout this section, we shall assume that $f$ satisfies the hypotheses of the Commutator Theorem, i.e., $f \in I_{\alpha}(b)$ for some function $b \in \mathrm{BMO}$. In particular, this implies that $f \in I_{\alpha / 2}(\mathrm{BMO})$, so that the operator $K_{\alpha}$, given by

$$
K_{\alpha} g(t)=\int_{-\infty}^{\infty} \frac{(f(s)-f(t))^{2}}{|s-t|^{1+\alpha}} g(s) d s
$$

is actually bounded on $L^{p}, 1<p<\infty$. We shall often suppress the subscript, referring to $K_{\alpha}$ simply as $K$, and we shall denote the kernel of $K$ by $B_{2}(s, t)$; clearly $B_{2}(s, t)=|s-t|^{\alpha} A_{2}(s, t)$.

The main result of this section is

Theorem 1. For every $\alpha \in(0,1)$ and $p \in[2, \infty)$, there is a constant $c=c_{\alpha, p}$ such that

$$
\left\|K_{\alpha} g\right\|_{\dot{B}_{p p}^{\alpha}} \leq c\|b\|_{*}^{2}\|g\|_{p}
$$

for all $g \in L^{p}(\mathfrak{R})$ and $b \in \mathrm{BMO}$ such that $f=I_{\alpha}(b)$.

The proof of Theorem 1 relies upon the Commutator Theorem together with the following elementary lemma: 
Lemma 3.1. For all $\gamma, M_{0}>0$, there is a constant $c=c_{\gamma, M_{0}}$ such that

$$
\begin{aligned}
& \int_{0}^{M_{0} h} u^{\gamma-1}|F(u+t+h)| d u \leq c h^{\gamma} F^{*}(t+h), \\
& \int_{M_{0} h}^{\infty} u^{-\gamma-1}|F(u+t+h)| d u \leq c h^{-\gamma} F^{*}(t+h)
\end{aligned}
$$

for every $t \in \mathfrak{R}, h>0$, and $F \in L_{\mathrm{loc}}^{1}(\mathfrak{R})$, where $F^{*}$ denotes the HardyLittlewood maximal function of $F$.

Proof of Lemma 3.1. For each integer $k$, let $J_{k}=\left[2^{-k-1} M_{0} h, 2^{-k} M_{0} h\right]$. Then

$$
\int_{0}^{M_{0} h} u^{\gamma-1}|F(u+t+h)| d u=\sum_{k=0}^{\infty} \int_{J_{k}} u^{\gamma-1}|F(u+t+h)| d u
$$

and

$$
\int_{M_{0} h}^{\infty} u^{-\gamma-1}|F(u+t+h)| d u=\sum_{-\infty}^{k=-1} \int_{J_{k}} u^{-\gamma-1}|F(u+t+h)| d u
$$

Now

$$
\begin{aligned}
\int_{J_{k}} u^{\gamma-1}|F(u+t+h)| d u & \leq c\left(2^{-k} h\right)^{\gamma-1} \int_{2^{-(k+1)} M_{0} h}^{2^{-k} M_{0} h}|F(u+t+h)| d u \\
& \leq c 2^{-\gamma k} h^{\gamma} F^{*}(t+h)
\end{aligned}
$$

and

$$
\begin{aligned}
\int_{J_{k}} u^{-\gamma-1}|F(u+t+h)| d u & \leq c\left(2^{-k} h\right)^{-\gamma-1} \int_{2^{-(k+1)} M_{0} h}^{2^{-k} M_{0} h}|F(u+t+h)| d u \\
& \leq c 2^{\gamma k} h^{-\gamma} F^{*}(t+h)
\end{aligned}
$$

(3.3) follows from (3.5) and (3.7), while (3.4) follows similarly from (3.6) and (3.8).

To begin the proof of Theorem 1, we write

$$
\begin{aligned}
K g(t+h)-K g(t) & =\int_{-\infty}^{\infty} B_{2}(s, t+h) g(s) d s-\int_{-\infty}^{\infty} B_{2}(s, t) g(s) d s \\
& =\theta_{1}(t, h)+\theta_{2}(t, h)+\theta_{3}(t, h)
\end{aligned}
$$

where

$$
\begin{aligned}
& \theta_{1}(t, h)=-\int_{|s-t|<100 h} B_{2}(s, t) g(s) d s, \\
& \theta_{2}(t, h)=\int_{|s-t|<100 h} B_{2}(s, t+h) g(s) d s, \\
& \theta_{3}(t, h)=\int_{|s-t|>100 h}\left\{B_{2}(s, t+h)-B_{2}(s, t)\right\} g(s) d s .
\end{aligned}
$$


For $j=1,2,3$, we shall estimate

$$
\int_{\mathfrak{R}} \int_{0}^{\infty}\left|\theta_{j}(t, h)\right|^{p} h^{-1-\alpha p} d h d t .
$$

We begin with

Lemma 3.2. For every $\alpha \in(0,1)$ and for all $p \in(1, \infty)$ there is a constant $c=c_{\alpha, p}$ such that, for $j=1$ or 2 ,

$$
\int_{\mathfrak{R}} \int_{0}^{\infty}\left|\theta_{j}(t, h)\right|^{p} h^{-1-\alpha p} d h d t \leq c_{\alpha, p}\|b\|_{*}^{2 p}\|g\|_{p}^{p} .
$$

Proof of Lemma 3.2. Let $\chi=\chi_{[0,1]}$. Then

$$
\begin{aligned}
& \int_{\mathfrak{R}} \int_{0}^{\infty}\left|\theta_{1}(t, h)\right|^{p} h^{-1-\alpha p} d h d t \\
& \quad \leq \int_{\mathfrak{R}} \int_{0}^{\infty}\left\{\int_{\mathfrak{R}} \chi\left(\frac{|s-t|}{100 h}\right) B_{2}(s, t)|g(s)| d s\right\}^{p} h^{-1-\alpha p} d h d t \\
& \quad \leq \int_{\mathfrak{R}}\left\{\int_{\mathfrak{R}} B_{2}(s, t)|g(s)|\left(\int_{0}^{\infty} \chi\left(\frac{|s-t|}{100 h}\right) h^{-1-\alpha p} d h\right)^{1 / p} d s\right\}^{p} d t
\end{aligned}
$$

by the Minkowski integral inequality. Moreover,

$$
\begin{aligned}
& \left(\int_{0}^{\infty} \chi\left(\frac{|s-t|}{100 h}\right) h^{-1-\alpha p} d h\right)^{1 / p} \\
& \quad=\left(\int_{|s-t| / 100}^{\infty} h^{-1-\alpha p} d h\right)^{1 / p}=c_{\alpha, p}|s-t|^{-\alpha},
\end{aligned}
$$

so that

$$
\begin{aligned}
\int_{\mathfrak{R}} \int_{0}^{\infty}\left|\theta_{1}(t, h)\right|^{p} h^{-1-\alpha p} d h d t & \leq c_{\alpha, p} \int_{\mathfrak{R}}\left\{\int_{\mathfrak{R}} A_{2}(s, t)|g(s)| d s\right\}^{p} d t \\
& =c_{\alpha, p}\left\|C_{2}(|g|)\right\|_{p}^{p} \leq c_{\alpha, p}\|b\|_{*}^{2 p}\|g\|_{p}^{p}
\end{aligned}
$$

by the Commutator Theorem, with $k=2$.

We turn next to $\theta_{2}$. For $h>0$ and $t \in \mathfrak{R}$, a change of variables in (3.11) yields

$$
\begin{aligned}
\left|\theta_{2}(t, h)\right| & \leq \int_{|u|<101 h} B_{2}(u+t+h, t+h)|g(u+t+h)| d u \\
& \leq c_{\alpha}\|b\|_{*}^{2} \int_{|u|<101 h} u^{\alpha-1}|g(u+t+h)| d u \\
& \leq c_{\alpha}\|b\|_{*}^{2} h^{\alpha} g^{*}(t+h)
\end{aligned}
$$

by (3.3) and the fact that the $\operatorname{Lip}_{\alpha}$ norm of $f$ is dominated by the BMO norm of $b$. Now let $q=p /(p-1)$ be the conjugate exponent to $p$. By (3.18), we 
have

$$
\begin{aligned}
& \int_{\mathfrak{R}} \int_{0}^{\infty}\left|\theta_{2}(t, h)\right|^{p} h^{-1-\alpha p} d h d t \\
& \leq c_{\alpha, p}\|b\|_{*}^{2(p-1)} \int_{\mathfrak{R}} \int_{0}^{\infty}\left[h^{\alpha} g^{*}(t+h)\right]^{p-1} \\
& \quad \times\left\{\int_{|u|<101 h} B_{2}(u+t+h, t+h)|g(u+t+h)| d u\right\} h^{-1-\alpha p} d h d t \\
& =c_{\alpha, p}\|b\|_{*}^{2 p / q} \int_{\mathfrak{R}} \int_{0}^{\infty}\left\{\int_{|u|<101 h} B_{2}(u+t+h, t+h)|g(u+t+h)| d u\right\} \\
& \times c_{\alpha, p}\|b\|_{*}^{2 p / q} \int_{\mathfrak{R}} \int_{w}^{\infty}\left\{\int_{|u|<101(\xi-w)} B_{2}(t+h+u, 2 \xi)|g(2 \xi+u)| d u\right\} \\
& \times \frac{g^{*}(2 \xi)^{p / q}}{(\xi-w)^{1+\alpha}} d \xi d w d t
\end{aligned}
$$

where, in the last equality, we have made the change of variables $\xi=\frac{1}{2}(t+h)$, $w=\frac{1}{2}(t-h)$. The rightmost expression in (3.19) equals

$$
\begin{aligned}
c_{\alpha, p}\|b\|_{*}^{2 p / q} \int_{\mathfrak{R}} \int_{\mathfrak{R}} \int_{\mathfrak{R}} B_{2}(2 \xi+ & u, 2 \xi)|g(2 \xi+u)| g^{*}(2 \xi)^{p / q} \\
& \times \chi\left(\frac{|u|}{101(\xi-w)}\right)(\xi-w)^{-1-\alpha} d u d \xi d w \\
=c_{\alpha, p}\|b\|_{*}^{2 p / q} \int_{\mathfrak{R}} & \int_{\mathfrak{R}} B_{2}(2 \xi+u, 2 \xi)|g(2 \xi+u)| g^{*}(2 \xi)^{p / q} \\
\times & \left\{\int_{\mathfrak{R}} \chi\left(\frac{|u|}{101(\xi-w)}\right)(\xi-w)^{-1-\alpha} d w\right\} d u d \xi .
\end{aligned}
$$

Now we have

$$
\int_{\mathfrak{R}} \chi\left(\frac{|u|}{101(\xi-w)}\right)(\xi-w)^{-1-\alpha} d w=\int_{|u| / 101}^{\infty} \lambda^{-1-\alpha} d \lambda=c_{\alpha}|u|^{-\alpha}
$$

so that, by $(3.19)-(3.21)$,

$$
\begin{aligned}
& \int_{\mathfrak{R}} \int_{0}^{\infty}\left|\theta_{2}(t, h)\right|^{p} h^{-1-\alpha p} d h d t \\
& \quad \leq c_{\alpha, p}\|b\|_{*}^{2 p / q} \int_{\mathfrak{R}}\left\{\int_{\mathfrak{R}} A_{2}(2 \xi+u, 2 \xi)|g(2 \xi+u)| d u\right\} g^{*}(2 \xi)^{p / q} d \xi \\
& \quad=c_{\alpha, p}\|b\|_{*}^{2 p / q} \int_{\mathfrak{R}} C_{2}(|g|)(2 \xi) \cdot g^{*}(2 \xi)^{p / q} d \xi \\
& \quad \leq c_{\alpha, p}\|b\|_{*}^{2 p / q}\left\|C_{2}(|g|)\right\|_{p}\left\|g^{*}\right\|_{p}^{p / q} \leq c_{\alpha, p}\|b\|_{*}^{2 p}\|g\|_{p}^{p}
\end{aligned}
$$

by Hölder's inequality, the Commutator Theorem, and the $L^{p}$-boundedness of the Hardy-Littlewood maximal operator.

The estimate for $\theta_{3}$ requires a restriction upon the range of $p$ : 
Lemma 3.3. For every $\alpha \in(0,1)$ and for all $p \in[2, \infty)$ the estimate (3.14) continues to hold with $j=3$.

Proof of Lemma 3.3. We begin by writing

$$
B_{2}(s, t+h)-B_{2}(s, t)=\sigma_{1}(s, t+h)+\sigma_{2}(s, t, h),
$$

where

$$
\begin{aligned}
& \sigma_{1}(s, t, h)=\left[|s-t-h|^{-1-\alpha}-|s-t|^{-1-\alpha}\right](f(s)-f(t+h))^{2} \\
& \sigma_{2}(s, t, h)=|s-t|^{-1-\alpha}\left[(f(s)-f(t+h))^{2}-(f(s)-f(t))^{2}\right] .
\end{aligned}
$$

For $h>0,|s-t|>100 h$, an application of the mean-value theorem yields (3.26) $\left|\sigma_{1}(s, t, h)\right| \leq c_{\alpha} h|s-t-h|^{-2-\alpha}(f(s)-f(t+h))^{2} \leq c_{\alpha}\|b\|_{*}^{2} h|s-t-h|^{\alpha-2}$ whence

$$
\begin{aligned}
& \int_{|s-t|>100 h}\left|\sigma_{1}(s, t, h) \| g(s)\right| d s \\
& \quad \leq c_{\alpha}\|b\|_{*}^{2} \int_{|s-t|>100 h} h|s-t-h|^{\alpha-2}|g(s)| d s \\
& \quad \leq c_{\alpha}\|b\|_{*}^{2} h \int_{|u|>99 h} u^{\alpha-2}|g(u+t+h)| d u \leq c_{\alpha}\|b\|_{*}^{2} h^{\alpha} g^{*}(t+h)
\end{aligned}
$$

by (3.4) with $\gamma=1-\alpha$. Raising (3.27) to the power $p-1=p / q$ and combining this with (3.26) yields

$$
\begin{array}{r}
\int_{\mathfrak{R}} \int_{0}^{\infty}\left(\int_{|s-t|>100 h}\left|\sigma_{1}(s, t, h) \| g(s)\right| d s\right)^{p} h^{-1-\alpha p} d h d t \\
\leq c_{\alpha, p}\|b\|_{*}^{2 p / q} \int_{\mathfrak{R}} \int_{0}^{\infty}\left\{\int_{|s-t|>100 h} \frac{(f(s)-f(t+h))^{2}}{|s-t-h|^{\alpha+2}}|g(s)| d s\right\} \\
\leq c_{\alpha, p}\|b\|_{*}^{2 p / q} \int_{\mathfrak{R}} \int_{0}^{\infty}\left\{\int_{|u|>99 h} B_{2}(t+h+u, t+h) u^{-1}|g(u+t+h)| d u\right\} \\
\times g^{*}(t+h)^{p / q} h^{-\alpha} d h d t .
\end{array}
$$

As before, the change of variables $\xi=\frac{1}{2}(t+h), w=\frac{1}{2}(t-h)$ transforms this last expression into

$$
\begin{aligned}
c_{\alpha, p}\|b\|_{*}^{2 p / q} \int_{\mathfrak{R}} \int_{w}^{\infty}\left\{\int_{|u|>99(\xi-w)} B_{2}(2 \xi+u, 2 \xi) u^{-1}|g(2 \xi+u)| d u\right\} \\
\times \frac{g^{*}(2 \xi)^{p / q}}{(\xi-w)^{\alpha}} d \xi d w \\
=c_{\alpha, p}\|b\|_{*}^{2 p / q} \int_{\mathfrak{R}} \int_{\mathfrak{R}} B_{2}(2 \xi+u, 2 \xi) u^{-1}|g(2 \xi+u)| g^{*}(2 \xi)^{p / q} \\
\times\left\{\int_{-\infty}^{\xi}\left[1-\chi\left(\frac{|u|}{99(\xi-w)}\right)\right](\xi-w)^{-\alpha} d w\right\} d u d \xi
\end{aligned}
$$


(compare (3.19), (3.20)). Now we have

$$
\begin{aligned}
\int_{-\infty}^{\xi} & {\left[1-\chi\left(\frac{|u|}{99(\xi-w)}\right)\right](\xi-w)^{-\alpha} d w } \\
= & \int_{\xi-|u| / 99}^{\xi}(\xi-w)^{-\alpha} d w=c_{\alpha}|u|^{1-\alpha}
\end{aligned}
$$

so that (3.28)-(3.30) imply

$$
\begin{aligned}
& \int_{\mathfrak{R}} \int_{0}^{\infty}\left(\int_{|s-t|>100 h}\left|\sigma_{1}(s, t, h) \| g(s)\right| d s\right)^{p} h^{-1-\alpha p} d h d t \\
& \quad \leq c_{\alpha, p}\|b\|_{*}^{2 p / q} \int_{\mathfrak{R}}\left\{\int_{\mathfrak{R}} A_{2}(2 \xi+u, 2 \xi)|g(2 \xi+u)| d u\right\} g^{*}(2 \xi)^{p / q} d \xi \\
& \quad \leq c_{\alpha, p}\|b\|_{*}^{2 p}\|g\|_{p}^{p}
\end{aligned}
$$

just as in (3.22). Note that the derivation of (3.31) did not actually require that $p \geq 2$, only that $p \in(1, \infty)$.

Turning to $\sigma_{2}$, we have

$$
\sigma_{2}(s, t, h)=|s-t|^{-1-\alpha}(f(t)-f(t+h))[2(f(s)-f(t))+(f(t)-f(t+h))]
$$

so that

$$
\begin{aligned}
\int_{|s-t|>100 h} \sigma_{2}(s, t, h) g(s) d s= & 2(f(t)-f(t+h)) C_{1,100 h} g(t) \\
& +(f(t)-f(t+h))^{2} T_{\alpha, 100 h} g(t),
\end{aligned}
$$

where $C_{1,100 h}$ is the truncation defined by $(2.7)$, and

$$
T_{\alpha, 100 h} g(t)=\int_{|s-t|>100 h}|s-t|^{-1-\alpha} g(s) d s .
$$

We claim that the nontangential maximal function

$$
\sup _{\left|t-t_{0}\right|<h} h^{\alpha}\left|T_{\alpha, 100 h} g(t)\right| \text { is in } L^{p}
$$

with norm bounded by $c_{\alpha, p}\|g\|_{p}$. This follows from (3.4) once we observe that, for $\left|t-t_{0}\right|<h$,

$$
h^{\alpha}\left|T_{\alpha, 100 h} g(t)\right| \leq c_{\alpha} h^{\alpha} \int_{|u|>95 h}|u|^{-1-\alpha}\left|g\left(u+t_{0}\right)\right| d u \leq c_{\alpha} g^{*}\left(t_{0}\right) .
$$

From this, it follows that

$$
\begin{array}{r}
\int_{\mathfrak{R}} \int_{0}^{\infty}\left|(f(t)-f(t+h))^{2} T_{\alpha, 100 h} g(t)\right|^{p} h^{-1-\alpha p} d h d t \\
\leq c_{\alpha, p}\|b\|_{*}^{2 p / q} \int_{\mathfrak{R}} \int_{0}^{\infty}\left|h^{\alpha} T_{\alpha, 100 h} g(t)\right|^{p} d \mu(t, h)
\end{array}
$$

where $d \mu$ is the Carleson measure given by (2.5). By Carleson's theorem (see, e.g., [T, Chapter 15, Theorem 1.1]), (3.37) is dominated by $c_{\alpha, p}\|b\|_{*}^{2 p}\|g\|_{p}^{p}$. 
Again, the estimate (3.37) is valid for $p \in(1, \infty)$. It is the estimate of the first part of the right-hand side of (3.33) that imposes the restriction upon $p$.

We claim that the nontangential maximal function

$$
\sup _{\left|t-t_{0}\right| \leq h}\left|C_{1,100 h} g(t)\right| \text { is in } L^{p}
$$

with norm bounded by $c_{\alpha, p}\|b\|_{*}\|g\|_{p}$. To see this, observe that, by (2.11)$(2.12)$,

$$
\begin{aligned}
\left|C_{1,100 h} g(t)\right| \leq & \left|\int_{|s-t|>100 h}\left(A_{1}(s, t)-A_{1}\left(s, t_{0}\right)\right) g(s) d s\right| \\
& +\left|\int_{|s-t|>100 h} A_{1}\left(s, t_{0}\right) g(s) d s\right| \\
\leq & c_{\alpha}\|b\|_{*} h^{\alpha} \int_{\left|s-t_{0}\right|>99 h}\left|s-t_{0}\right|^{-1-\alpha}|g(s)| d s+\left|C_{1,100 h}(g)\left(t_{0}\right)\right| \\
& +c_{\alpha}\|b\|_{*} \int_{90 h<\left|s-t_{0}\right|<100 h}\left|s-t_{0}\right|^{-1}|g(s)| d s
\end{aligned}
$$

whenever $\left|t-t_{0}\right|<h$. Applying (3.4), the first and third terms are bounded by $c_{\alpha}\|b\|_{*} g^{*}\left(t_{0}\right)$, while $\left|C_{1,100 h}(g)\left(t_{0}\right)\right|$ is dominated by $C_{1}^{*} g\left(t_{0}\right)$. Thus we obtain (3.38) with the desired estimate by an application of (2.9). From this it follows that, for $p \geq 2$,

$$
\begin{aligned}
& \int_{\mathfrak{R}} \int_{0}^{\infty}\left|2(f(t)-f(t+h)) C_{1,100 h} g(t)\right|^{p} h^{-1-\alpha p} d h d t \\
& \quad \leq c_{\alpha, p}\|b\|_{*}^{p-2} \int_{\mathfrak{R}} \int_{0}^{\infty}\left|C_{1,100 h} g\left(t_{0}\right)\right|^{p} d \mu(t, h) \leq c_{\alpha, p}\|b\|_{*}^{2 p}\|g\|_{p}^{p}
\end{aligned}
$$

by Carleson's theorem. The lemma now follows on combining (3.31), (3.32), the estimate for (3.37), and (3.40).

Theorem 1 now follows from Lemma 3.2 and Lemma 3.3. We remark that the proof of Theorem 1 may be extended with minor modifications to the operator obtained from $K_{\alpha}$ by replacing the kernel $B_{2}(s, t)$ by $B_{2}(s, t) \chi_{(0, \infty)}(s-t)$. From this it follows that Theorem 1 holds for the operator with kernels $B_{2}(s, t) \chi_{(-\infty, 0)}(s-t)$ and $B_{2}(s, t) \operatorname{sgn}(s-t)$. Moreover, one can show that the operator obtained by multiplying any of these kernels by $(f(s)-f(t))^{2 k}|s-t|^{-\alpha k}, k \in N$, maps $L^{p}$ to $\dot{B}_{p p}^{\alpha}$ with constant no worse than $(k+1) c_{\alpha, p}\|b\|_{*}^{2}\|f\|_{\text {Lip }_{\alpha}}^{2 k}$, when $p \geq 2$. With this in view, consider the modified single-layer potential operator $\mathscr{S}_{\alpha}$, given by

$$
\mathscr{S}_{\alpha} g(t)=\int_{\mathfrak{R}} W_{\alpha}(s, t) g(s) d s,
$$

where

$$
W_{\alpha}(s, t)=|s-t|^{\alpha-1} \exp \left[-\frac{(f(s)-f(t))^{2}}{|s-t|^{2 \alpha}}\right]
$$


For $p \in[2, \infty), \alpha \in(0,1), f \in I_{\alpha}(\mathrm{BMO})$, there is a constant $c=c_{p, \alpha, f}$ such that

$$
\left\|\mathscr{S}_{\alpha} g\right\|_{\dot{B}_{p p}^{\alpha}} \leq c\|g\|_{L^{p}}
$$

To see this, observe that

$$
W_{\alpha}(s, t) g(s)=|s-t|^{\alpha-1} g(s)+\sum_{k=0}^{\infty} \frac{(-1)^{k+1}}{(k+1) !} \frac{(f(s)-f(t))^{k+1}}{|s-t|^{1+\alpha+2 \alpha k}} g(s) ;
$$

thus $\mathscr{S}_{\alpha} g(t)$ differs from a constant times $I_{\alpha} g(t)$ by a convergent series of operators mapping $L^{p}$ to $\dot{B}_{p p}^{\alpha}$. Since $I_{\alpha}$ maps $L^{p}$ to $\dot{B}_{p p}^{\alpha}$ for $p \geq 2$ we obtain the desired estimate. But we can actually do a little better:

Theorem 2. For $p \in[2, \infty), \alpha \in(0,1)$, there is a constant $c=c_{\alpha, p}$, such that

$$
\left\|\mathscr{S}_{\alpha} g\right\|_{\dot{B}_{p p}^{\alpha}} \leq c\left(1+\|b\|_{*}^{4}\right)\|g\|_{p}
$$

for all $f=I_{\alpha}(b), b \in \mathrm{BMO}$. Moreover, this result continues to hold when the kernel of $\mathscr{S}_{\alpha}^{\alpha}$ is multiplied by $\chi_{(0, \infty)}(s-t), \chi_{(-\infty, 0)}(s-t)$, or $\operatorname{sgn}(s-t)$.

Proof. $\mathscr{S}_{\alpha} g(t)$ differs from a constant times $I_{\alpha} g(t)$ by the operator

$$
\mathscr{L} g(t)=\int_{\mathfrak{R}} L(s, t) g(t) d t,
$$

where

$$
L(s, t)=|s-t|^{\alpha-1}\left\{\exp \left[-\frac{f(s)-f(t)}{|s-t|^{2 \alpha}}\right]-1\right\} .
$$

Since, for $x \in \mathfrak{R}, 0 \leq 1-e^{-|x|} \leq|x|$, we see that $|L(s, t)| \leq B_{2}(s, t)$. Now we may write $\mathscr{L} g(t+h)-\mathscr{L} g(t)$ as $\left(\theta_{1}+\theta_{2}+\theta_{3}\right)(t, h)$ as in (3.9)-(3.12), with $L$ in place of $B_{2}$. In this context, Lemma 3.2 continues to hold. To prove the analogue of Lemma 3.3, we write, as in (3.23)-(3.25),

$$
L(s, t+h)-L(s, t)=\sigma_{1}(s, t, h)+\sigma_{2}(s, t, h),
$$

where

$$
\begin{array}{r}
\sigma_{1}(s, t, h)=\left[|s-t-h|^{\alpha-1}-|s-t|^{\alpha-1}\right]\left\{\exp \left[-\frac{(f(s)-f(t+h))^{2}}{|s-t-h|^{2 \alpha}}\right]-1\right\} \\
\sigma_{2}(s, t, h)=|s-t|^{\alpha-1}\left\{\exp \left[-\frac{(f(s)-f(t+h))^{2}}{|s-t-h|^{2 \alpha}}\right]\right. \\
\left.-\exp \left[-\frac{f(s)-f(t))^{2}}{|s-t|^{2 \alpha}}\right]\right\}
\end{array}
$$

It is easy to see that $\sigma_{1}$ continues to satisfy (3.26), and that (3.49)

$$
\sigma_{2}(s, t, h)=|s-t|^{\alpha-1}\left\{\frac{(f(s)-f(t))^{2}}{|s-t|^{2 \alpha}}-\frac{(f(s)-f(t+h))^{2}}{|s-t-h|^{2 \alpha}}\right\}(1+E(s, t, h))
$$


where $E(s, t, h)$ is an error term which is bounded in absolute value by $\|f\|_{\text {Lip }_{\alpha}}^{2}$. A straightforward modification of the proof of Lemma 3.3 now yields (3.44).

Corollary 3.4. For $p \in[2, \infty), f=I_{1 / 2}(b), b \in \mathrm{BMO}$, the boundary singlelayer potential operator $\mathscr{S}^{b}$ defined by (1.3) maps $L^{p}$ into $\dot{B}_{p p}^{\alpha}$, and there is a constant $c_{p}$ independent of $f, g$ such that

$$
\left\|\mathscr{S}^{b} g\right\|_{\dot{B}_{p p}^{\alpha}} \leq c_{p}\left(1+\|b\|_{*}^{4}\right)\|g\|_{p} .
$$

\section{Formal INVERSiON OF the Riesz POTENTIAL}

In this section we prove

Theorem 3. For every $\alpha \in(0,1), p \in(1, \infty)$, and $g \in L^{p}(\mathfrak{R})$, there exists $\gamma \in L^{p}(\Re)$ such that $K_{\alpha} g=I_{\alpha} \gamma$.

We remark that in the case $p=2$, Theorem 3 is immediate from Theorem 1 since $\dot{B}_{22}^{\alpha}=I_{\alpha}\left(L^{2}\right)$. To prove Theorem 3 for general $p$, we shall construct a formal inverse $D^{\alpha}$ for $I_{\alpha}$, and we shall show that the operator $D^{\alpha} K_{\alpha}$ maps $L^{p}$ boundedly into $L^{p}$; from this we will be able to deduce Theorem 3 .

For $\delta>\varepsilon>0$ and $\phi \in \mathscr{S}$, define

$$
\begin{aligned}
D_{\varepsilon, \delta}^{\alpha} \phi(t)= & \int_{\varepsilon<|h|<\delta}(\phi(t+h)-\phi(t))|h|^{-1-\alpha} d h, \\
& D^{\alpha} \phi(t)=\lim _{\substack{\varepsilon \rightarrow 0 \\
\delta \rightarrow \infty}} D_{\varepsilon, \delta}^{\alpha} \phi(t) .
\end{aligned}
$$

Straightforward calculation shows that $\left(D^{\alpha} \phi\right)^{\wedge}(\xi)=c_{\alpha}|\xi|^{\alpha} \hat{\phi}(\xi)$, so that $D^{\alpha}$ is formally inverse to $I_{\alpha}$. Let $S=S_{\varepsilon, \delta}$ be the operator $D_{\varepsilon, \delta}^{\alpha} K_{\alpha}$, and denote by $S^{0}$ the principal-value operator $D^{\alpha} K_{\alpha}$. We shall show, first of all, that $S_{\varepsilon, \delta}$ is bounded on $L^{p}$ uniformly in $\varepsilon$ and $\delta$, and shall then use this to deduce the $L^{p}$ boundedness of $S^{0}$. We remark first of all that it can be shown that $S^{0}$ need not map $L^{\infty}$ boundedly into BMO; a proof of this fact is outlined in $\S 5$. Consequently, the T1 Theorem is not helpful; hence the seemingly primitive approach taken here.

In a fashion analogous to that in the proof of Theorem 1, we write $S$ as the sum of five operators $S_{1}, S_{2}, S_{3}, S_{4}, S_{5}$, where for $p \in(1, \infty)$ and $g \in L^{p}$,

$$
\begin{gathered}
S_{1} g(t)=\int_{\varepsilon<|h|<\delta}\left\{\int_{|s-t|<100|h|}-B_{2}(s, t) g(s) d s\right\}|h|^{-1-\alpha} d h \\
S_{2} g(t)=\int_{\varepsilon<|h|<\delta}\left\{\int_{|s-t|<100|h|} B_{2}(s, t, h) g(s) d s\right\}|h|^{-1-\alpha} d h \\
S_{3} g(t)=\int_{\varepsilon<|h|<\delta}\left\{\int_{|s-t|>100|h|}\left[|s-t-h|^{-1-\alpha}-|s-t|^{-1-\alpha}\right]\right. \\
\left.\times(f(s)-f(t+h))^{2} g(s) d s\right\}|h|^{-1-\alpha} d h
\end{gathered}
$$




$$
\begin{aligned}
S_{4} g(t)=\int_{\varepsilon<|h|<\delta}\left\{\int_{|s-t|>100|h|}(f(t)-\right. & f(t+h))^{2} \\
& \left.\times|s-t|^{-1-\alpha} g(s) d s\right\}|h|^{-1-\alpha} d h
\end{aligned}
$$

$$
\begin{array}{r}
S_{5} g(t)=2 \int_{\varepsilon<|h|<\delta}\left\{\int_{|s-t|>100|h|} \frac{(f(s)-f(t))}{|s-t|^{1+\alpha}} g(s) d s\right\} \\
\times(f(t)-f(t+h))|h|^{-1-\alpha} d h .
\end{array}
$$

Note that $S_{1} g, S_{2} g, S_{3} g$, and $S_{4} g$ are integrals involving $\theta_{1}, \theta_{2}, \sigma_{1}$, and the 'good part' of $\sigma_{2}$, respectively, while $S_{5} g$ is an integral involving that part of $\sigma_{2}$ which was most troublesome in the proof of Lemma 3.3 (cf. (3.10)-(3.12), (3.23)-(3.25)). Not surprisingly, the $L^{p}$ estimates for $S_{1}$ through $S_{4}$ will be fairly routine, while the estimate for $S_{5}$ is more problematic.

For $1 \leq j \leq 4$, let $V_{j}(|g|)$ denote the integral obtained from $S_{j} g$ by replacing the inmost integrand in (4.2)-(4.5) by its absolute value and letting $\varepsilon=0$, $\delta=\infty$. Then we have

Lemma 4.1. For every $\alpha \in(0,1)$ and for all $p \in(1, \infty)$, there is constant $c_{\alpha, p}$ such that, for $1 \leq j \leq 4,\left\|V_{j}(|g|)\right\|_{p} \leq c_{\alpha, p}\|b\|_{*}^{2}\|g\|_{p}$.

Proof. Let $q$ be the conjugate exponent to $p$, and suppose $\psi \in L^{q}$. Then

$$
\begin{aligned}
& \left|\int_{\mathfrak{R}} V_{1}(|g|)(t) \psi(t) d t\right| \\
& \quad \leq \int_{\mathfrak{R}} \int_{\mathfrak{R}} \int_{|s-t|<100|h|} B_{2}(s, t)|g(s)||h|^{-1-\alpha}|\psi(t)| d s d h d t \\
& \quad \leq c_{\alpha} \int_{\mathfrak{R}}\left\{\int_{\mathfrak{R}} A_{2}(s, t)|g(s)| d s\right\}|\psi(t)| d t \leq c_{\alpha, p}\|b\|_{*}^{2}\|\psi\|_{q}\|g\|_{p}
\end{aligned}
$$

by the Commutator Theorem. Furthermore,

$$
\begin{aligned}
\mid \int_{\mathfrak{R}} & V_{2}(|g|)(t) \psi(t) d t \mid \\
& \leq \int_{\mathfrak{R}} \int_{\mathfrak{R}} \int_{|u|<101|h|} B_{2}(u+t+h, t+h)|g(u+t+h)||h|^{-1-\alpha}|\psi(t)| d u d t d h \\
& =\int_{\mathfrak{R}} \int_{\mathfrak{R}}\left\{\int_{\mathfrak{R}} \chi\left(\frac{|u|}{101|\xi-w|}\right)|\psi(\xi+w)||\xi-w|^{-1-\alpha} d w\right\} \\
& \times B_{2}(2 \xi+u, 2 \xi)|g(2 \xi+u)| d u d \xi \\
\leq & c_{\alpha} \iint_{\mathfrak{R}^{2}} A_{2}(2 \xi+u, 2 \xi)|g(2 \xi+u)| \psi^{*}(2 \xi) d u d \xi \leq c_{\alpha, p}\|b\|_{*}^{2}\|\psi\|_{q}\|g\|_{p}
\end{aligned}
$$

using the change of variables $\xi=\frac{1}{2}(t+h), w=\frac{1}{2}(t-h)$ in by now familiar fashion, together with Lemma 3.1, the Commutator Theorem, and the HardyLittlewood Maximal Theorem (compare (3.18)-(3.21)). 
Likewise, we have

$$
\begin{aligned}
& \left|\int_{\mathfrak{R}} V_{3}(|g|)(t) \psi(t) d t\right| \\
& \quad \leq c_{\alpha} \iint_{\mathfrak{R}^{2}}\left\{\int_{|u|>99|h|}|u|^{-1} B_{2}(u+t+h, t+h)|g(u+t+h)| d u\right\} \\
& \quad=c_{\alpha} \iint_{\mathfrak{R}^{2}}\left\{\int_{\mathfrak{R}}\left[1-\chi\left(\frac{|u|}{99|\xi-w|}\right)\right]|\xi-w|^{-\alpha}|\psi(t)| d h d t\right. \\
& \quad \leq c_{\alpha} \iint_{\mathfrak{R}^{2}} A_{2}(2 \xi+u, 2 \xi) \psi^{*}(2 \xi)|g(2 \xi+u)| d u d \xi \\
& \quad \leq c_{\alpha, p}\|b\|_{*}^{2}\|\psi\|_{q}\|g\|_{p}
\end{aligned}
$$

(compare (3.27)-(3.31)). Finally

$$
\begin{aligned}
& \left|\int_{\mathfrak{R}} V_{4}(|g|)(t) \psi(t) d t\right| \\
& \quad \leq \iint_{\mathfrak{R}^{2}}\left\{\int_{|u|>100|h|}|u|^{-1-\alpha}|g(u+t)| d u\right\} B_{2}(t+h, t)|\psi(t)| d h d t \\
& \quad \leq c_{\alpha} \iint_{\mathfrak{R}^{2}} A_{2}(t+h, t)\left|g^{*}(t+h)\right| \psi(t) \mid d h d t \leq c_{\alpha, p}\|b\|_{*}^{2}\|\psi\|_{q}\|g\|_{p}
\end{aligned}
$$

using a simple change of variables and Lemma 3.1.

For $l \leq j \leq 5$, denote by $S_{j}^{0} g$ the principal-value integral obtained by taking the pointwise limit as $\varepsilon \rightarrow 0, \delta \rightarrow \infty$ of $S_{j} g$. Then we have

Corollary 4.2. Let $\alpha \in(0,1), p \in(1, \infty), g \in L^{p}$, and $1 \leq j \leq 4$. Then $S_{j}^{0} g$ exists pointwise almost everywhere and in $L^{p}$; moreover, there is a constant $c_{\alpha, p}$ independent of $g$ such that

$$
\left\|S_{j}^{0} g\right\|_{p} \leq c_{\alpha, p}\|b\|_{*}^{2}\|g\|_{p}
$$

Furthermore, if $g \in L^{2}$, then $S_{5}^{0} g$ exists in $L^{2}$, and (4.11) is satisfied with $j=5, p=2$.

Proof. For $1 \leq j \leq 4,\left|S_{j} g\right|$ is dominated pointwise by $V_{j}(|g|)$. Standard arguments using the Lebesgue dominated convergence theorem establish the existence of $S_{j}^{0} g$ pointwise a.e. and in $L^{p}$, with the desired estimate. Since Theorem 3 is true when $p=2$ by Theorem 1 , we know the existence of $S^{0} g$ pointwise a.e. and in $L^{2}$ provided $g$ is in $L^{2}$, with the desired estimate.

Since $S_{5}^{0}=S^{0}-\sum_{j=1}^{4} S_{j}^{0}$, we obtain the $L^{2}$ result for $S_{5}^{0}$ (in roundabout fashion). 
To complete the proof of Theorem 3, we must obtain (4.1) for $j=5$ and $1<p<\infty$. To begin, we obtain a weak-type $(1,1)$ estimate for $S_{5}$ which is uniform in $\varepsilon$ and $\delta$.

Lemma 4.3. There is a constant $c_{\alpha}$ independent of $\varepsilon$ and $\delta$ such that for every $\lambda>0$ and for all $g \in L^{1}$,

$$
\lambda\left|\left\{x:\left|S_{5} g(x)\right|>\lambda\right\}\right| \leq c_{\alpha}\|b\|_{*}^{2}\|g\|_{p} .
$$

Proof. Using the standard technique of Calderon-Zygmund decomposition into good and bad functions, and our $L^{2}$ estimates, it suffices to prove that if $\beta$ is an $L^{1}$ function supported in $(-\lambda, \lambda)$ with integral zero, then

$$
\int_{|t|>100 \lambda}\left|S_{5} \beta(t)\right| d t \leq c_{\alpha}\|b\|_{*}^{2}\|\beta\|_{1}
$$

(see [St] or [T]). We begin by writing $S_{5} \beta=N_{1} \beta+N_{2} \beta$, where for $j=1,2$,

$$
\begin{aligned}
N_{j} \beta(t) & =2 \int_{\varepsilon_{j}<|h|<\delta_{j}} A_{1}(t, t+h) C_{1,100|h|} \beta(t) d h \\
& =2 \int_{\varepsilon_{j}<|h|<\delta_{j}} A_{1}(t, t+h) \int_{|s-t|>100|h|} A_{1}(s, t) \beta(s) d s d h
\end{aligned}
$$

with

$$
\begin{array}{ll}
\varepsilon_{1}=\min \{\varepsilon, \lambda / 2\}, & c_{2}=\max \{\varepsilon, \lambda / 2\}, \\
\delta_{1}=\min \{\delta, \lambda / 2\}, & \delta_{2}=\max \{\delta, \lambda / 2\}
\end{array}
$$

(cf. (2.7), (2.10), (4.6)). It is easy to see that $N_{1}=0$ unless $\varepsilon_{1}=\varepsilon$ and $N_{2}=0$ unless $\delta_{2}=\delta$. Moreover for $|t|>100 \lambda, C_{1,100|h|} \beta(t)$ is nonzero only if $|h|<(\lambda+|t|) / 100$. let

With all this in mind, we estimate $N_{2} \beta(t)$. Let $X=\left\{h: \varepsilon_{2}<|h|<\delta_{2}\right\}$ and

$$
F_{3}=\left(\frac{-\lambda+|t|}{100}, \frac{\lambda+|t|}{100}\right), \quad F_{4}=\left(\frac{-\lambda-|t|}{100}, \frac{-\lambda+|t|}{100}\right) .
$$

We write $N_{2} \beta(t)=N_{3} \beta(t)+N_{4} \beta(t)$, where $N_{3} \beta(t)$ (resp. $N_{4} \beta(t)$ ) is that piece of $N_{2} \beta(t)$ arising from integration with respect to $h$ over $X \cap F_{3}$ (resp. $\left.X \cap F_{4}\right)$. Now, for $|t|>100 \lambda$, we have

$$
\int_{|s-t|>100|h|}\left|A_{1}(s, t) \beta(s)\right| d s \leq c_{\alpha}\|b\|_{*}|h|^{-1}\|\beta\|_{1}, \quad \text { for } h \in F_{3} \cap X
$$

using the standard estimate (2.11) for $A_{1}$. A second application of (2.11) yields

$$
\left|N_{3} \beta(t)\right| \leq c_{\alpha}\|b\|_{*}^{2}\|\beta\|_{1} \int_{F_{3} \cap X}|h|^{-2} d h .
$$

Now, for $h \in F_{3} \cap X,|t|>100 \lambda$, we have $c|t|>(\lambda+|t|) / 100 \geq|h|$; moreover, $\left|F_{3} \cap X\right| \leq c \lambda$. Thus

$$
\left|N_{3} \beta(t)\right| \leq c_{\alpha}\|b\|_{*}^{2}\|\beta\| \|_{1} \lambda|t|^{-2}, \quad|t|>100 \lambda .
$$


Integrating (4.17), we have

$$
\int_{|t|>100 \lambda}\left|N_{3} \beta(t)\right| d t \leq c_{\alpha}\|b\|_{*}^{2}\|\beta\|_{1} \int_{|t|>100 \lambda}\left(\frac{|t|}{\lambda}\right)^{-2} \frac{d t}{\lambda} \leq c_{\alpha}\|b\|_{*}^{2}\|\beta\|_{1} .
$$

Turning to $N_{4} \beta$, we observe first of all that $h \in F_{4}$ if and only if $100|h|+\lambda<$ $|t|$; hence whenever $h \in F_{4} \cap X$ and $|s| \leq \lambda$, we have $|s-t| \geq|t|-|s|>100|h|$. Consequently, since $\int \beta=0$, we obtain

$$
N_{4} \beta(t)=2 \int_{F_{4} \cap X} A_{1}(t, t+h) \int_{|s| \leq \lambda}\left[A_{1}(s, t)-A_{1}(0, t)\right] \beta(s) d s d h .
$$

By (2.11) and (2.12), we have, for $|t|>100 \lambda$,

$$
\begin{aligned}
\left|N_{4} \beta(t)\right| & \leq c_{\alpha}\|b\|_{*}^{2} \int_{F_{4} \cap X}|h|^{-1} \int_{|s| \leq \lambda}|s|^{\alpha}|t|^{-1-\alpha}|\beta(s)| d s d h \\
& \leq c_{\alpha}\|b\|_{*}^{2}\|\beta\|_{1} \lambda^{\alpha}|t|^{-1-\alpha} \int_{\lambda / 2<|h|<|t| / 100}|h|^{-1} d h \\
& =c_{\alpha}\|b\|_{*}^{2}\|\beta\|_{1} \lambda^{\alpha}|t|^{-1-\alpha} \ln \left(\frac{|t|}{50 \lambda}\right) .
\end{aligned}
$$

Thus

(4.21)

$$
\int_{|t|>100 \lambda}\left|N_{4} \beta(t)\right| d t \leq c_{\alpha}\|b\|_{*}^{2}\|\beta\|_{1} \int_{|t|>100 \lambda}\left(\frac{\lambda}{|t|}\right)^{1+\alpha} \ln \left(\frac{|t|}{\lambda}\right) d t=c_{\alpha}\|b\|_{*}^{2}\|\beta\|_{1} .
$$

Combining (4.18) and (4.21), we obtain (4.13) with $N_{2}$ in place of $S_{5}$.

Next we consider $N_{1} \beta$. If $|s| \leq \lambda,|t|>100 \lambda$, and $|h|<\delta_{1} \leq \lambda / 2$, then we have $|s-t| \geq|t|-|s|>99 \lambda>100|h|$. Thus by (4.14), we actually have

$$
N_{1} \beta(t)=2 C_{1} \beta(t) \int_{\varepsilon_{j}<|h|<\delta_{j}} A_{1}(t, t+h) d h=-2 C_{1} \beta(t)\left(C_{1 ; \varepsilon_{j}, \delta_{j}} 1\right)(t),
$$

where $C_{1 ; \varepsilon_{j}, \delta_{j}}$ denotes the doubly-truncated version of the commutator $C_{1}$.

Since $\int \beta=0$, we have

$$
C_{1} \beta(t)=\int_{|s| \leq \lambda}\left[A_{1}(s, t)-A_{1}(0, t)\right] \beta(s) d s .
$$

Moreover, for $|s| \leq \lambda$ and $|t|>100 \lambda$, (2.12) yields

$$
\left|A_{1}(s, t)-A_{1}(0, t)\right| \leq c_{\alpha}\|b\|_{*} \frac{\lambda^{\alpha}}{|t|^{1+\alpha}}
$$

so that

$$
\left|C_{1} \beta(t)\right| \leq c_{\alpha}\|b\|_{*}\|\beta\|_{1} \frac{\lambda^{\alpha}}{|t|^{1+\alpha}} \text { for }|t|>100 \lambda .
$$

For ease of notation, let us write $T^{\lambda}=C_{1 ; \varepsilon_{1}, \delta_{1}} 1$; since $C_{1}$ is a CalderónZygmund operator, $T^{\lambda}$ is in BMO with $\left\|T^{\lambda}\right\|_{*} \leq c_{\alpha}\|b\|_{*}$. Let $T_{\mathrm{av}}^{\lambda}$ denote the 
mean of $T^{\lambda}$ on $(-\lambda, \lambda)$. Then, letting $X=\left\{h: \varepsilon_{1}<|h|<\delta_{1}\right\}$, we have

$$
\begin{aligned}
\left|T_{\mathrm{av}}^{\lambda}\right| & =\left.\frac{1}{2 \lambda}\left|\int_{X}\left\{\int_{-\lambda}^{\lambda}[(f(t+h)-f(0))+(f(0)-f(t))] d t\right\}\right| h\right|^{-1-\alpha} d h \mid \\
& =\left.\frac{1}{2 \lambda}\left|\int_{X}\left\{\int_{\lambda}^{\lambda+h}(f(t)-f(0)) d t+\int_{-\lambda+h}^{-\lambda}(f(t)-f(0)) d t\right\}\right| h\right|^{-1-\alpha} d h \mid \\
& \leq \frac{1}{2 \lambda} \int_{|h| \leq \lambda / 2} c_{\alpha}\|b\|_{*}|h|^{-\alpha} \lambda^{\alpha} d h=c_{\alpha}\|b\|_{*} .
\end{aligned}
$$

Then, by (4.25) and (4.26),

$$
\begin{aligned}
\int_{|t|>100 \lambda}\left|N_{1} \beta(t)\right| d t \leq & 2 \int_{|t|>100 \lambda}\left|C_{1} \beta(t) \| T^{\lambda}(t)-T_{\mathrm{av}}^{\lambda}\right| d t \\
& +2 \int_{|t|>100 \lambda}\left|T_{\mathrm{av}}^{\lambda} \| C_{1} \beta(t)\right| d t \\
\leq & c_{\alpha}\|b\|\left\|_{*}\right\| \beta \|_{1}\left\{\int_{|t|>100 \lambda}\left|T^{\lambda}(t)-T_{\mathrm{av}}^{\lambda}\right| \frac{\lambda^{\alpha}}{|t|^{1+\alpha}} d t\right. \\
& \left.\quad+\|b\|_{*} \int_{|t|>100 \lambda} \frac{\lambda^{\alpha}}{|t|^{1+\alpha}} d t\right\} .
\end{aligned}
$$

By a theorem of C. Fefferman (see [Str, Lemma 2.2] for a proof), the first term in the curly brackets is less than or equal to $\left\|T^{\lambda}\right\|_{*}$, which is in turn less than or equal to $c_{\alpha}\|b\|_{*}$; the second term in curly brackets is exactly equal to $c_{\alpha}\|b\|_{*}$. Thus (4.27) yields (4.13) with $N_{1}$ in place of $S_{5}$. This completes the proof of (4.13).

By standard arguments, Corollary 4.2 and Lemma 4.3 show that $S_{5}$ is of strong-type $(2,2)$ and of weak-type $(1,1)$, so that by interpolation, $S_{5}^{0}$ is of strong-type $(p, p)$ for $1<p \leq 2$. To obtain results for $p>2$, it is natural to try to prove a weak-type $(1,1)$ result for the adjoint $\widetilde{S}_{5}^{0}$. Unfortunately, a straightforward adaptation of the proof of Lemma 4.3 fails for the adjoint-as, indeed, it should! For, if we could prove (4.13) for $\widetilde{S}_{5}$, we would easily be able to extend this result to a proof that $\widetilde{S}_{5}^{0}$ maps $H^{1}$ atoms into $L^{1}$, and hence that $S_{5}^{0}$ maps $L^{\infty}$ to BMO. But this last statement is false, since $S_{5}^{0}$ is the "bad part" of $S^{0}$, and $S^{0}$ need not map $L^{\infty}$ to BMO. Thus we proceed along a more circuitous route.

We introduce some additional notation. Up until now we have suppressed the dependence of $S_{5}$ upon $\varepsilon$ and $\delta$; hereafter we emphasize it by writing $S_{5}^{\ell, \delta}$. When $\delta=\infty$ the operator will be denoted by $S_{5}^{\varepsilon}$. The maximal operator $S_{5}^{*}$ is defined for $g \in L^{1} \cap L^{2}$ and $t \in \mathfrak{R}$ by

$$
S_{5}^{*} g(t)=\sup _{\varepsilon>0}\left|S_{5}^{\varepsilon} g(t)\right| \text {. }
$$

We write $M g$ in place of $g^{*}$ to denote the Hardy-Littlewood maximal function of $g ; M^{2} g$ denotes $M(M g)$. 
We begin our journey with

Lemma 4.4. There is a constant $c_{\alpha}>0$ such that for all $\lambda>0$ and for all $t, t_{0}$ with $\left|t-t_{0}\right|<\lambda$,

$$
\left|S_{5}^{2 \lambda} g(t)-S_{5}^{2 \lambda} g\left(t_{0}\right)\right| \leq c_{\alpha}\|b\|_{*}\left\{\|b\|_{*} M^{2} g\left(t_{0}\right)+C_{0}^{*} g\left(t_{0}\right)\right\}
$$

whenever $g \in L^{1} \cap L^{2}$.

Proof. By (4.6),

$$
\begin{aligned}
& \frac{1}{2}\left[S_{5}^{2 \lambda} g(t)-S_{5}^{2 \lambda} g\left(t_{0}\right)\right] \\
& =\int_{2 \lambda<|h|}\left[C_{1,100|h|} g(t) A_{1}(t, t+h)-C_{1,100|h|} g\left(t_{0}\right) A_{1}\left(t_{0}, t_{0}+h\right)\right] d h \\
& =\int_{2 \lambda<|h|}\left\{C_{1,100|h|} g(t)-C_{1,100|h|} g\left(t_{0}\right)\right\} A_{1}(t, t+h) d h \\
& \quad+\int_{2 \lambda<|h|} C_{1,100|h|} g\left(t_{0}\right)\left\{A_{1}(t, t+h)-A_{1}\left(t_{0}, t_{0}+h\right)\right\} d h .
\end{aligned}
$$

For $\left|t-t_{0}\right|<\lambda$ and $2 \lambda<|h|$, clearly

$$
\begin{aligned}
& \left.\mid A_{1}(t), t+h\right)-A_{1}\left(t_{0}, t_{0}+h\right) \mid \\
& \quad \leq|h|^{-(1+\alpha)}\left(\left|f(t)-f\left(t_{0}\right)\right|+\left|f(t+h)-f\left(t_{0}+h\right)\right|\right) \\
& \quad \leq c_{\alpha}\|b\|_{*}|h|^{-1-\alpha} \lambda^{\alpha}
\end{aligned}
$$

so that

(4.32) $\int_{2 \lambda<|h|}\left|C_{1,100|h|} g\left(t_{0}\right)\left\|A_{1}(t, t+h)-A_{1}\left(t_{0}, t_{0}+h\right) \mid d h \leq c_{\alpha}\right\| b \|_{*} C_{1}^{*} g\left(t_{0}\right)\right.$.

Moreover,

(4.33) $C_{1,100|h|} g(t)-C_{1,100|h|} g\left(t_{0}\right)$

$$
=\int_{|s-t|>100|h|}\left(A_{1}(s, t)-A_{1}\left(s, t_{0}\right)\right) g(s) d s+\int_{\mathscr{S}} A_{1}\left(s, t_{0}\right) g(s) d s
$$

where, for $\left|t-t_{0}\right|<\lambda$,

$$
\begin{aligned}
\mathscr{S} & =\left\{s:|s-t| \leq 100|h|<\left|s-t_{0}\right|\right\} \\
& = \begin{cases}\left(t_{0}+100|h|, t+100|h|\right), & t>t_{0}, \\
\left(t-100|h|, t_{0}-100|h|\right), & t<t_{0} .\end{cases}
\end{aligned}
$$

Consequently

$$
\begin{aligned}
\int_{\mathscr{S}}\left|A_{1}\left(s, t_{0}\right) \| g(s)\right| d s & \leq c_{\alpha}\|b\|_{*}\left|t-t_{0}\right| \frac{1}{|\mathscr{S}|} \int_{\mathscr{S}}\left|s-t_{0}\right|^{-1}|g(s)| d s \\
& \leq c_{\alpha}\|b\|_{*} \lambda|h|^{-1} M g\left(t_{0}+\operatorname{sgn}\left(t-t_{0}\right) \cdot 100|h|\right) .
\end{aligned}
$$


Thus by Lemma 3.1 and (2.11),

$$
\begin{aligned}
\int_{2 \lambda<|h|} & \left|A_{1}(t, t+h)\right| \int_{\mathscr{S}}\left|A_{1}\left(s, t_{0}\right) \| g(s)\right| d s \\
& \leq c_{\alpha}\|b\|_{*}^{2} \lambda \int_{2 \lambda<|h|}|h|^{-2}\left[M g\left(t_{0}-100|h|\right)+M g\left(t_{0}+100|h|\right)\right] d h \\
& \leq c_{\alpha}\|b\|_{*}^{2} M^{2} g\left(t_{0}\right) .
\end{aligned}
$$

Now, for $|s-t|>100|h|,\left|t-t_{0}\right|<\lambda$, and $2 \lambda<|h|,(2.12)$ yields

$$
\left|A_{1}(s, t)-A_{1}\left(s, t_{0}\right)\right| \leq c_{\alpha}\|b\|_{*} \lambda^{\alpha}\left|s-t_{0}\right|^{-1-\alpha}
$$

so that

$$
\begin{aligned}
& \int_{|s-t|>100|h|}\left|A_{1}(s, t)-A_{1}\left(s, t_{0}\right) \| g(s)\right| d s \\
& \leq c_{\alpha}\|b\|_{*} \lambda^{\alpha} \int_{\left|s-t_{0}\right| \geq 99|h|}\left|s-t_{0}\right|^{-1-\alpha}|g(s)| d s \\
& \leq c_{\alpha}\|b\|_{*} \lambda^{\alpha}|h|^{-\alpha} M g\left(t_{0}\right)
\end{aligned}
$$

and

$$
\text { (4.39) } \begin{gathered}
\int_{2 \lambda<|h|}\left|A_{1}(t, t+h)\right| \int_{|s-t|>100|h|}\left|A_{1}(s, t)-A_{1}\left(s, t_{0}\right)\right||g(s)| d s d h \\
\leq c_{\alpha}\|b\|_{*}^{2} \lambda^{\alpha} M g\left(t_{0}\right) \int_{2 \lambda<|h|}|h|^{-1-\alpha} d h \leq c_{\alpha}\|b\|_{*}^{2} M^{2} g\left(t_{0}\right)
\end{gathered}
$$

using (2.11) and the fact that $M g \leq M^{2} g$ pointwise. Combining (4.30), (4.32), (4.33), (4.36), and (4.39) yields (4.29).

We shall also require the following result, a bit of a potpourri:

Lemma 4.5. Let $\lambda>0$, let $g \in L^{1} \cap L^{2}$, suppose $t_{0} \in \mathfrak{R}$, and write $g=g_{1}+g_{2}$, where $g_{1}$ is the restriction of $g$ to $\left[t_{0}-800 \lambda, t_{0}+800 \lambda\right]$. Then

$$
\begin{gathered}
-\frac{1}{2} S_{5}^{0,2 \lambda} g_{2}(t)=C_{1} g_{2}(t)\left(C_{1 ; 0,2 \lambda} 1\right)(t) \quad \text { for }\left|t-t_{0}\right|<\lambda ; \\
\left|C_{1} g_{2}(t)-C_{1} g_{2}\left(t_{0}\right)\right| \leq c_{\alpha}\|b\|_{*} M g\left(t_{0}\right) \quad \text { for }\left|t-t_{0}\right|<\lambda ; \\
\left|C_{1} g_{2}\left(t_{0}\right)\right| \leq C_{1}^{*} g\left(t_{0}\right) ; \\
\int_{t_{0}-\lambda}^{t_{0}+\lambda}\left|\left(C_{1 ; 0,2 \lambda} 1\right)(t)\right| d t \leq \lambda c_{\alpha}\|b\|_{*} .
\end{gathered}
$$

Proof. We obtain (4.40) and (4.43) for essentially the same reasons as (4.22) and (4.26) in the proof of Lemma 4.3. The estimate (4.42) is obvious, while (4.41) follows easily from the standard estimate (2.12) for $A_{1}$.

Next, we combine Lemmas 4.4 and 4.5 to prove the following result, based on an idea of Cotlar (cf. [T, pp. 291-293]): 
Lemma 4.6. There is a constant $c=c_{\alpha}$ such that for all $g \in L^{1} \cap L^{2}$ and for all $t_{0} \in \mathfrak{R}$,

$$
S_{5}^{*} g\left(t_{0}\right) \leq c\left\{\left[M\left(\left|S_{5}^{0} g\right|^{1 / 2}\right)\left(t_{0}\right)\right]^{2}+\|b\|_{*}\left(C_{1}^{*} g\right)\left(t_{0}\right)+\|b\|_{*}^{2}\left(M^{2} g\right)\left(t_{0}\right)\right\} .
$$

Proof. The proof is by contradiction: we assume that for each positive constant $c$, there is a function $g \in L^{1} \cap L^{2}$ and a point $t_{0} \in \mathfrak{R}$ such that (4.44) fails. The idea is to choose $c$ so large that we obtain an absurdity. We know that there is a positive $\lambda$ for which

$$
\left|S_{5}^{2 \lambda} g\left(t_{0}\right)\right| \geq \frac{1}{2} S_{5}^{*} g\left(t_{0}\right)=\rho,
$$

so that we have for $c>20$,

$$
C_{1}^{*} g\left(t_{0}\right)+\|b\|_{*} M^{2} g\left(t_{0}\right) \leq \frac{\rho}{c\|b\|_{*}}, \quad\left[M\left(\left|S_{5}^{0} g\right|^{1 / 2}\right)\left(t_{0}\right)\right]^{2} \leq \frac{\rho}{20} .
$$

The second inequality in (4.46) forces the existence of a subinterval $F$ of $\left(t_{0}-\right.$ $\left.\lambda, t_{0}+\lambda\right)$ which contains $t_{0}$ such that $|F| \geq \lambda / 4$ and $\left|S_{5}^{0} g(t)\right| \leq \rho / 10$ for all $t \in F$. From this fact, together with Lemma 4.4 and the first inequality in (4.46), we deduce that

$$
-S_{5}^{0,2 \lambda} g(t) \geq \rho / 2, \quad t \in F .
$$

Now let $g=g_{1}+g_{2}$ as in Lemma 4.5. By (4.47), there is a measurable set $F_{1} \subseteq F$ such that $\left|F_{1}\right| \geq \lambda / 8$ and such that either $(\mathrm{A})-S_{5}^{0,2 \lambda} g_{1}(t) \geq \rho / 4$ for all $t \in F_{1}$, or $(\mathrm{B})-S_{5}^{0,2 \lambda} g_{2}(t) \geq \rho / 4$ for all $t \in F_{1}$.

In case (A), Lemma 4.3 and the first inequality in (4.46) yield

$$
\lambda / 8 \leq\left|F_{1}\right| \leq c_{\alpha}\|b\|_{*}^{2}\left\|g_{1}\right\|_{1} \rho^{-1} \leq 1600 c_{\alpha} \lambda \rho^{-1} M g\left(t_{0}\right)\|b\|_{*}^{2}<\lambda / 8,
$$

for $c$ large enough, a contradiction. In case (B), (4.40) of Lemma 4.5 yields

$$
\left|C_{1} g_{2}(t)\left(C_{1 ; 0,2 \lambda} 1\right)(t)\right| \geq \rho / 2, \quad t \in F_{1} .
$$

However, (4.41) and (4.42) of Lemma 4.5 show that, for $\left|t-t_{0}\right|<\lambda$,

$$
\left[c_{\alpha}\|b\|_{*} M g\left(t_{0}\right)+C_{1}^{*} g\left(t_{0}\right)\right]\left|C_{1 ; 0,2 \lambda} 1(t)\right| \geq\left|C_{1} g_{2}(t)\left(C_{1 ; 0,2 \lambda}\right) 1(t)\right| .
$$

Combining (4.46), (4.49), and (4.50), we have

$$
c_{\alpha} \rho\left|C_{1 ; 0,2 \lambda} 1(t)\right| \geq \rho c\|b\|_{*}, \quad t \in F_{1},
$$

so that

$$
\lambda c\|b\|_{*} /\left(8 c_{\alpha}\right) \leq \int_{t_{0}-\lambda}^{t_{0}+\lambda}\left|\left(C_{1 ; 0,2 \lambda} 1\right)(t)\right| d t,
$$

contradicting (4.43) of Lemma 4.5, for $c$ large. In both cases we obtain a contradiction, so the result is true.

We use Lemma 4.6 to prove a good $\lambda$-inequality: 
Lemma 4.7. For every $\rho>0$ there is a constant $c_{\rho}=c_{\rho, \alpha}$ such that, for all $\lambda>0$ and for all $g \in L^{1} \cap L^{2}$,

$$
\begin{aligned}
\left|\left\{S_{5}^{*} g>2 \lambda\right\}\right| \leq & \rho\left|\left\{S_{5}^{*} g>\lambda\right\}\right| \\
& +2\left|\left\{\|b\|_{*}^{2} M^{2} g>c_{\rho} \lambda\right\}\right|+2\left|\left\{\|b\|_{*} C_{1}^{*} g>c_{\rho} \lambda\right\}\right| .
\end{aligned}
$$

Proof. Let $E=E_{\lambda, \rho}$ denote the set $\left\{\|b\|_{*}^{2} M^{2} g>c_{\rho} \lambda\right\} \cup\left\{\|b\|_{*} C_{1}^{*} g>c_{\rho} \lambda\right\}$. For the moment we allow $c_{\rho}$ to vary, but later we will fix it at a value satisfying several conditions. Since $\mathscr{S}_{\lambda}=\left\{S_{5}^{*} g>\lambda\right\}$ is an open set of finite Lebesgue measure, it admits a Whitney decomposition, i.e., there is a family $\left\{I_{j}: j \in N\right\}$ of closed intervals having pairwise disjoint interiors such that $\mathscr{S}_{\lambda}$ is equal to the union of the $I_{j}$ 's and such that $\left|I_{j}\right| \sim \operatorname{dist}\left(I_{j}, \mathscr{S}_{\lambda}^{c}\right)$ with constants independent of $j$ (cf. [St] or [T]). Let $\sigma_{1}=\sigma_{1, \lambda, \rho}$ be the set of all $j$ for which $I_{j} \subseteq E_{\lambda}$, and let $\sigma_{2}$ be the complement of $\sigma_{1}$ in the positive integers. Now

$$
\left|\bigcup_{j \in \sigma_{1}} I_{j}\right| \leq\left|E_{\lambda}\right|
$$

while for each $j \in \sigma_{2}$, there exists $t_{0} \in I_{j}$ with $t_{0} \notin E_{\lambda}$, i.e.,

$$
\|b\|_{*} M^{2} g\left(t_{0}\right)+C_{1}^{*} g\left(t_{0}\right) \leq 2 c_{\rho} \lambda /\|b\|_{*} .
$$

Suppose $j \in \sigma_{2}$ and write $I_{j}=I=\left[t_{2}-\gamma, t_{2}+\gamma\right]$. Since $|I| \sim \operatorname{dist}\left(I, \mathscr{S}_{\lambda}^{c}\right)$, there exists $t_{1} \in \mathscr{S}_{\lambda}^{c}$ with, say, $\left|t_{1}-t_{2}\right| \leq 20 \gamma$; hence $S_{5}^{*} g\left(t_{1}\right) \leq \lambda$. Now, if $\varepsilon \geq 100 \gamma, t \in I$, and $t_{0} \in I$ satisfies (4.55), then we deduce exactly as in the proof of Lemma 4.4 that

$$
\left|S_{5}^{\varepsilon} g(t)-S_{5}^{\varepsilon} g\left(t_{1}\right)\right| \leq c_{\alpha}\|b\|_{*}\left\{\|b\|_{*} M^{2} g\left(t_{0}\right)+C_{1}^{*} g\left(t_{0}\right)\right\} \leq 2 c_{\alpha}\left(c_{\rho} \lambda\right) \leq \lambda / 4
$$

if $c_{\rho}$ is chosen small enough.

Now, let $t \in I \cap \mathscr{S}_{2 \lambda}$, and write $g=g_{1}+g_{2}$, where $g_{1}$ is the restriction of $g$ to $\left[t_{2}-800 \gamma, t_{2}+800 \gamma\right]=800 I$. We claim that either $(\mathrm{A}) S_{5}^{*} g_{1}(t)>\lambda / 4$, or (B) there exists $\eta=\eta(t) \in(0,100 \gamma)$ with $\left|S_{5}^{\eta, 100 \gamma} g_{2}(t)\right|>\lambda / 4$. For, if (A) does not hold, then $S_{5}^{*} g_{2}(t)>7 \lambda / 4$, so there exists $\eta=\eta(t) \in(0,100 \gamma)$ with $\left|S_{5}^{\eta} g_{2}(t)\right|>7 \lambda / 4$; then by $(4.56)$

$$
\begin{aligned}
\left|S_{5}^{\eta, 100 \gamma} g_{2}(t)\right| \geq & \left|S_{5}^{\eta} g_{2}(t)\right|-\left|S_{5}^{100 \gamma} g(t)-S_{5}^{100 \gamma} g\left(t_{1}\right)\right| \\
& -\left|S_{5}^{100 \gamma} g\left(t_{1}\right)\right|-\left|S_{5}^{100 \gamma} g_{1}(t)\right| \\
> & 7 \lambda / 4-\lambda / 4-\lambda-\lambda / 4=\lambda / 4 .
\end{aligned}
$$

We begin by considering case (A). Now, either $t \in E_{\lambda}$, or else, as in (4.55),

$$
\|b\|_{*} M^{2} g_{1}(t)+C_{1}^{*} g_{1}(t) \leq 2\left(\|b\|_{*} M^{2} g(t)+C_{1}^{*} g(t)\right) \leq 4 c_{\rho} \lambda /\|b\|_{*}
$$

so that, by (4.44) applied to $g_{1}$,

$$
\lambda / 4<S_{5}^{*} g_{1}(t) \leq c\left[M\left(\left|S_{5}^{0} g_{1}\right|^{1 / 2}\right)(t)\right]^{2}+4 c_{\rho} c \lambda .
$$


If $c_{\rho}$ is small enough, we have, for $t \notin E_{\lambda}$,

$$
\lambda / 8<c\left[M\left(\left|S_{5}^{0} g_{1}\right|^{1 / 2}\right)(t)\right]^{2} .
$$

By Lemma 4.3, (4.12), with $S_{5}^{0}$ in place of $S_{5}$, we deduce that $\left|S_{5}^{0} g_{1}\right|^{1 / 2}$ is in weak- $L^{2}$, and in turn (by the usual covering argument) that $M\left(\left|S_{5}^{0} g_{1}\right|^{1 / 2}\right)$ is in weak- $L^{2}$ with

$$
\left|\left\{M\left(\left|S_{5}^{0} g_{1}\right|^{1 / 2}\right)>\varepsilon\right\}\right| \leq \varepsilon^{-2} c_{\alpha}\|b\|_{*}^{2}\|g\|_{1}
$$

for every $\varepsilon>0$. Thus, in particular, by (4.61) and (4.55),

$$
\begin{aligned}
& \left|\left\{\left[M\left(\left|S_{5}^{0} g_{1}\right|^{1 / 2}\right)(t)\right]^{2}>\lambda / 8 c\right\}\right| \leq 8 c c_{\alpha}\|b\|_{*}^{2}\left\|g_{1}\right\|_{1} \lambda^{-1} \\
& \quad \leq(1600)\left(8 c c_{\alpha}\|b\|_{*}^{2} M g_{1}\left(t_{0}\right) \gamma \lambda^{-1}\right) \leq(3200)\left(8 c c_{\alpha} c_{\rho}\right) \gamma \leq \gamma \rho / 2
\end{aligned}
$$

provided $c_{\rho}$ is chosen small enough. Thus, in case (A), we obtain

$$
\left|\left\{S_{5}^{*} g_{1}(t)>\lambda / 4\right\} \cap E_{\lambda}^{c}\right| \leq \gamma \rho / 2 .
$$

Turning now to case (B), we note first that, as in Lemma 4.5, (4.40),

$$
S_{5}^{\eta, 100 \gamma} g_{2}(t)=C_{1} g_{2}(t)\left(C_{1 ; \eta, 100 \gamma} 1\right)(t) \text {. }
$$

As in the proof of (4.50), we see that (arguing as in (4.41) and (4.42) of Lemma 4.5) since $t \in I$,

$$
\left[c_{\alpha}|| b \|_{*} M g\left(t_{0}\right)+C_{1}^{*} g\left(t_{0}\right)\right]\left|C_{1 ; \eta, 100 \gamma} 1(t)\right| \geq\left|S_{5}^{\eta, 100 \gamma} g_{2}(t)\right|>\lambda / 4 ;
$$

so, by (4.55),

$$
c_{\rho} c_{\alpha} \lambda\left|C_{1 ; \eta, 100 \gamma} 1(t)\right|>\left(\lambda|| b \|_{*}\right) / 4 .
$$

If we let $\psi$ denote the characteristic function of $100|I|=\left[t_{2}-100 \gamma, t_{2}+100 \gamma\right]$, a simple calculation involving the standard estimates for $A_{1}$ gives

$$
\left|C_{1 ; \eta, 100 \gamma} 1(t)\right| \leq C_{1}^{*} \psi(t)+c_{\alpha}\|b\|_{*} .
$$

For $c_{\rho}$ chosen small enough, (4.66) and (4.67) yield

$$
c_{\alpha} C_{1}^{*} \psi(t)>\|b\|_{*} c_{\rho}^{-1} .
$$

By the Commutator Theorem, $C_{1}^{*}$ is of strong-type $(2,2)$, so it is of weak-type $(2,2)$, and

$$
\left|\left\{C_{1}^{*} \psi(t)>\|b\|_{*}\left(c_{\rho} c_{\alpha}\right)^{-1}\right\}\right| \leq c_{\rho} c_{\alpha}\|\psi\|_{2}^{2}<\gamma \rho / 2,
$$

provided $c_{\rho}$ is small enough. So, in case (B), we have

$$
\left|\left\{\left|S_{5}^{\eta, 100 \gamma} g_{2}(t)\right|>\lambda / 4\right\} \cap E_{\lambda}^{c} \cap I\right| \leq \gamma \rho / 2 .
$$

Combining (4.63) and (4.70) yields

$$
\left|I \cap \mathscr{S}_{2 \lambda} \cap E_{\lambda}^{\mathcal{c}}\right| \leq \gamma \rho<|I| \rho .
$$


Thus

$$
\left|\bigcup_{j \in \sigma_{2}} I_{j} \cap \mathscr{S}_{2 \lambda} \cap E_{\lambda}^{c}\right|<\rho\left|\bigcup_{j \in \sigma_{2}} I_{j}\right| \leq \rho\left|\mathscr{S}_{\lambda}\right|
$$

so that by (4.54) and (4.72),

$$
\left|\mathscr{S}_{2 \lambda} \cap E_{\lambda}^{c}\right| \leq \rho\left|\mathscr{S}_{\lambda}\right|+\left|E_{\lambda}\right|
$$

because $\mathscr{S}_{2 \lambda} \subseteq \mathscr{S}_{\lambda}$. Thus $\left|\mathscr{S}_{2 \lambda}\right| \leq \rho\left|\mathscr{S}_{\lambda}\right|+2\left|E_{\lambda}\right|$, from which (4.53) follows.

Corollary 4.8. For every $p \in(1, \infty)$ and for all $g \in L^{1} \cap L^{2} \cap L^{p},\left\|S_{5}^{*} g\right\|_{p} \leq$ $c_{\alpha}\|b\|_{*}^{2}\|g\|_{p}$.

Proof. Let $R, \rho>0$. Then, by (4.53),

$$
\begin{aligned}
& 2^{-p} \int_{0}^{2 R} \lambda^{p-1}\left|\left\{S_{5}^{*} g>\lambda\right\}\right| d \lambda=\int_{0}^{R} \lambda^{p-1}\left|\left\{S_{5}^{*} g>2 \lambda\right\}\right| d \lambda \\
& \quad \leq \rho \int_{0}^{R} \lambda^{p-1}\left|\left\{S_{5}^{*} g>\lambda\right\}\right| d \lambda+2 \int_{0}^{\infty} \lambda^{p-1}\left|\left\{\|b\|_{*}^{2} M^{2} g>c_{\rho} \lambda\right\}\right| d \lambda \\
& \quad+2 \int_{0}^{\infty} \lambda^{p-1}\left|\left\{\|b\|_{*} C_{1}^{*} g>c_{\rho} \lambda\right\}\right| d \lambda \\
& \quad \leq \rho \int_{0}^{R} \lambda^{p-1}\left|\left\{S_{5}^{*} g>\lambda\right\}\right| d \lambda+\left(c_{\alpha}\|b\|_{*}^{2 p}\|g\|_{p}\right) /\left(c_{\rho}\right)^{p}
\end{aligned}
$$

by the Commutator Theorem and the Hardy-Littlewood Maximal Theorem. Now take $\rho=2^{-p-1}$, subtract, and let $R \rightarrow \infty$ to obtain the result.

Standard arguments involving the Dominated Convergence Theorem together with Corollaries 4.2 and 4.8 yield

Corollary 4.9. For $p \in(1, \infty), D^{\alpha} K_{\alpha}$ is bounded on $L^{p}$ with norm $\leq c_{\alpha, p}\|b\|_{*}^{2}$.

Theorem 3 now follows from Corollary 4.9; the function $\gamma$ is given by $c_{\alpha} D^{\alpha} K_{\alpha} g$. As a corollary, we also obtain

Theorem 4. For $p \in(1, \infty), \alpha \in(0,1), f=I_{\alpha}(b), b \in \mathrm{BMO}$, and $g \in$ $L^{p}(\mathfrak{R})$, there exists a function $\gamma \in L^{p}(\mathfrak{R})$ such that $\mathscr{S}_{\alpha} g=I_{\alpha}(\gamma)$, where $\mathscr{S}_{\alpha}$ is given by (3.41). Moreover, this result continues to hold when the kernel of $\mathscr{S}_{\alpha}^{\alpha}$ is multiplied by $\chi_{(0, \infty)}(s-t), \chi_{(-\infty, 0)}(s-t)$, or $\operatorname{sgn}(s-t)$.

Theorem 5. For $p \in(1, \infty), f=I_{1 / 2}(b), b \in \mathrm{BMO}$, and $g \in L^{p}(\Re)$, there exists a function $\gamma \in L^{p}(\Re)$ such that $\mathscr{S}^{b} g=I_{1 / 2}(\gamma)$, where $\mathscr{S}^{b}$ is the boundary single-layer potential operator given by (1.3). Moreover, given $\gamma \in L^{p}(\Re)$, there exists, for small $\|b\|_{*}, a g \in L^{p}(\mathfrak{R})$ with $\mathscr{S}^{b} g=I_{1 / 2}(\gamma)$.

The proof of Theorem 4 is a straightforward modification of the proof of Theorem 3, where now $K_{\alpha}$ is replaced by $\mathscr{S}_{\alpha}-c_{\alpha} I_{\alpha}$; the proof proceeds essentially along the same lines as the proof of Theorem 2 . For properly chosen $c_{\alpha}$ we get, using Corollary 4.9,

$$
\left\|\left(\mathscr{S}_{\alpha}^{b}-c_{\alpha} I_{\alpha}\right) g\right\|_{p} \leq c_{\alpha, p}\left(\|b\|_{*}^{2}+\|b\|_{*}^{4}\right) .
$$


The last part of Theorem 5 follows from expanding $\left(\mathscr{S}_{\alpha}^{b}\right)^{-1}$ in a Neumann series and using (4.75). We omit the details. Finally we remark that Theorem 5 is a one-dimensional analogue of Theorem 3.1 in [FR] for domains with time dependent boundaries.

\section{Appendix: The failure of The T1 TheOREM for $D^{\alpha} K_{\alpha}$}

In this section we outline a proof of the fact that the operator $S^{0}=D_{\alpha} K^{\alpha}$ need not map $L^{\infty}$ to BMO. We begin by defining the homogeneous Besov space $\dot{B}_{\infty 2}^{\alpha}$, the space of distributions $\phi \in S^{\prime}$ (modulo constants) satisfying

$$
\|\phi\|_{\dot{B}_{\infty 2}^{\alpha}}=\left(\int_{\mathfrak{R}}\left\|\Delta_{h} \phi\right\|_{\infty}^{2} h^{-1-2 \alpha} d h\right)^{1 / 2}<\infty
$$

where $\left(\Delta_{h} \phi\right)(t)=\phi(t+h)-\phi(t)$. The Dini class considered by Lewis and Silver in their work on the Dirichlet problem for the heat equation is essentially the subspace of functions in $\dot{B}_{\infty 2}^{1 / 2}$ having compact support (see [LeS]). It is easy to see that $\dot{B}_{\infty 2}^{\alpha}$ is properly contained in $I_{\alpha}(\mathrm{BMO})$ (see [Str]). We prove the following result:

Theorem 6. For every $\delta \in(0,1 / 2)$ there exists a function $f \in \dot{B}_{\infty 2}^{\alpha}$ such that for all $\varepsilon>0$ there is an interval $I$ with $|I|<\varepsilon$ satisfying

$$
\frac{1}{|I|} \int_{I}\left|\left(D^{\alpha} K_{\alpha} 1\right)(t)-m_{I}\left(D^{\alpha} K_{\alpha} 1\right)\right| d t \geq c_{f}\left(\log \frac{1}{|I|}\right)^{\delta} \text {. }
$$

Here, $m_{I}(\phi)=\frac{1}{|I|} \int_{I} \phi$.

Proof (sketch). It is easy to see that, for $f \in I_{\alpha}(\mathrm{BMO})$,

$$
\begin{aligned}
\left(D^{\alpha} K_{\alpha} 1\right)(t)= & \iint_{\mathfrak{R}^{2}} \frac{\left(\Delta_{u} \Delta_{h} f(t)\right)^{2}}{|u|^{1+\alpha}|h|^{1+\alpha}} d u d h \\
& -2 \iint_{\mathfrak{R}^{2}} \frac{\left(\Delta_{u} f\right)(t)\left(\Delta_{u} \Delta_{h} f\right)(t)}{|u|^{1+\alpha}|h|^{1+\alpha}} d u d h .
\end{aligned}
$$

For $f \in \dot{B}_{\infty 2}^{\alpha}$, it is a straightforward exercise to show that the first term on the right-hand side of $(5.2)$ is in $\operatorname{BMO}(\Re)$. It is then not hard to show that the second term on the right-hand side of $(5.2)$ is equal to

$$
-2 C_{1}\left(C_{1} 1\right)(t)+2\left[\left(C_{1} 1\right)(t)\right]^{2}
$$

Now, let $I$ be any interval on $\Re$, and let $\left(C_{1} 1\right)_{I}=m_{4 I}\left(C_{1} 1\right)$. We write

$$
\begin{aligned}
C_{1} 1 & =\left[C_{1} 1-\left(C_{1} 1\right)_{I}\right] \chi_{4 I}+\left[C_{1} 1-\left(C_{1} 1\right)_{I}\right] \chi_{\Re-4 I}+\left(C_{1} 1\right)_{I} \chi_{\Re} \\
& =g_{1}+g_{2}+g_{3} .
\end{aligned}
$$

By the Commutator Theorem, and the fact that $C_{1} 1 \in \mathrm{BMO}$, we have

$$
\int_{I}\left|C_{1} g_{1}(t)\right| d t \leq|I|^{1 / 2}|| C_{1} g_{1} \|_{2} \leq c_{f}|I|
$$


while the standard estimates for the kernel $A_{1}$ of $C_{1}$ and the fact that $C_{1} 1 \in$ BMO show

$$
\left|C_{1} g_{2}(t)-C_{1} g_{2}\left(t_{0}\right)\right| \leq c_{f}, \quad t, t_{0} \in I .
$$

Combining (5.4)-(5.6), we may write

$$
C_{1}\left(C_{1} 1\right)(t)=C_{1} g_{3}(t)+g_{4}(t)+m_{I}\left(C_{1} g_{2}\right)
$$

where $m_{I}\left(\left|g_{4}\right|\right) \leq c_{f}$. Now note that

$$
\begin{aligned}
& -2 C_{1} g_{3}(t)+2\left[\left(C_{1} 1\right)(t)\right]^{2} \\
& \quad=2\left[\left(C_{1} 1\right)(t)-\left(C_{1} 1\right)_{I}\right]^{2}+2\left(C_{1} 1\right)_{I}\left[\left(C_{1} 1\right)(t)-\left(C_{1} 1\right)_{I}\right] .
\end{aligned}
$$

Again, since $C_{1} 1 \in \mathrm{BMO}$, the first term on the right-hand side of (5.8) is in $L^{1}(I)$ with norm $\leq c_{f}|I|$. Combining (5.2)-(5.3), (5.7)-(5.8), and our discussion above, we see that, for $t \in I$,

$$
\left(D^{\alpha} K_{\alpha} 1\right)(t)=\phi(t)+2\left(C_{1} 1\right)_{I}\left[\left(C_{1} 1\right)(t)-\left(C_{1} 1\right)_{I}\right]
$$

with $\int_{I}\left|\phi-m_{I}(\phi)\right| d t \leq c_{f}|I|$.

Now let $\beta>1 / 2$ and define

$$
f(t)=f_{\alpha, \beta}(t)=\theta(t) \sum_{k=1}^{\infty} 2^{-k \alpha} k^{-\beta} \cos \left(2^{k} t\right),
$$

where $\theta$ is infinitely differentiable on $\Re$, and $\theta \equiv 1$ on $[-\pi, \pi]$ with $\operatorname{supp} \theta \subseteq$ $[-2 \pi, 2 \pi]$. By standard techniques for the estimation of lacunary series (cf. $[\mathrm{Z}$, Chapter II, $\S 4])$, we have $f \in L^{\infty}(\mathfrak{R})$ and

$$
\left|\Delta_{h} f(t)\right| \leq c|h|^{\alpha} \min \left\{1,\left[\log \left(\frac{1}{|h|}\right)\right]^{-\beta}\right\}
$$

in particular, it is easily seen that $f \in \dot{B}_{\infty 2}^{\alpha}$. Moreover, for $|h|,|t| \leq \frac{1}{4}$,

$$
\left|\Delta_{h}\left(M-C_{1} 1\right)(t)\right| \leq c|h|^{\alpha}, \quad\left|M(t)-\left(C_{1} 1\right)(t)\right| \leq c_{\alpha, \beta},
$$

where

$$
\begin{aligned}
M(t) & =\sum_{k=1}^{\infty} 2^{-k \alpha} k^{-\beta} \int_{\mathfrak{R}} \frac{\cos \left(2^{k}(t+h)\right)-\cos \left(2^{k} t\right)}{|h|^{1+\alpha}} d h \\
& =\mu_{\alpha} \sum_{k=1}^{\infty} k^{-\beta} \cos \left(2^{k} t\right), \quad \mu_{\alpha}=\int_{\mathfrak{R}} \frac{\cos u-1}{|u|^{1+\alpha}} d u .
\end{aligned}
$$

Now let $I=\left[0,2^{-l} \pi\right]$, where $l$ is a large positive integer. Writing

$$
M_{1}(t)=\mu_{\alpha} \sum_{k=1}^{l+4} k^{-\beta} \cos \left(2^{k} t\right), \quad M_{2}(t)=M(t)-M_{1}(t)
$$


we find, using standard techniques for the estimation of lacunary series,

$$
\begin{gathered}
m_{4 I}\left(M_{1}\right) \sim l^{1-\beta}, \quad m_{4 I}\left(M_{2}\right)=0, \\
m_{I}\left(\left|M_{2}\right|\right) \sim l^{(1 / 2-\beta)}, \quad m_{I}\left(M_{2}\right)=0, \\
m_{I}\left(\left|M_{1}-m_{I}\left(M_{1}\right)\right|\right) \leq c_{\alpha, \beta} l^{-\beta},
\end{gathered}
$$

where $\sim$ means the two quantities are proportional. By (5.14(ii), (iii)), we have

$$
\frac{1}{|I|} \int_{I}\left|M(t)-m_{I}(M)\right| d t \geq c_{\alpha, \beta} l^{(1 / 2-\beta)},
$$

so that by $(5.14(\mathrm{i}))$,

$$
\left|m_{4 I}(M)\right|\left(\frac{1}{|I|} \int_{I}\left|M(t)-m_{I}(M)\right| d t\right) \geq c_{\alpha, \beta} l^{(3 / 2-2 \beta)} .
$$

Consequently by (5.12), (5.14(i)), and (5.15),

$$
\begin{aligned}
& \left|\left(C_{1} 1\right)_{I}\right| \frac{1}{|I|} \int_{I}\left|\left(C_{1} 1\right)(t)-m_{I}\left(C_{1} 1\right)\right| d t \\
& \quad \geq\left[\left|m_{4 I}(M)\right|-c_{\alpha, \beta}\right]\left[\frac{1}{|I|} \int_{I}\left|M(t)-m_{I}(M)\right| d t-c_{\alpha, \beta} 2^{-l \alpha}\right] \\
& \quad \geq c_{\alpha, \beta} l^{(3 / 2-2 \beta)} \geq c_{\alpha, \beta}\left(\log \frac{1}{|I|}\right)^{(3 / 2-2 \beta)},
\end{aligned}
$$

provided $\beta \in(1 / 2,3 / 4)$. As $\beta$ ranges over this interval, $3 / 2-2 \beta$ ranges over $(0,1 / 2)$. Letting $\delta=3 / 2-2 \beta$ and using (5.9) we conclude that Theorem 6 follows from (5.17).

\section{REFERENCES}

[BL] J. Bergh and J. Lofstrom, Interpolation spaces: An introduction, Springer-Verlag, New York, 1976.

[Br1] R. M. Brown, The method of layer potentials for the heat equation in Lipschitz cylinders, Amer. J. Math. 111 (1989), 339-379.

[Br2] - The initial-Neumann problem for the heat equation in Lipschitz cylinders, Trans. Amer. Math. Soc. 320 (1990), 1-52.

[DJ] G. David and J.-L. Journé, A boundedness criterion for generalized Calderón-Zygmund operators, Ann. of Math. (2) 120 (1985), 371-397.

[FR] E. B. Fabes and N. M. Riviere, Dirichlet and Neumann problems for the heat equation in $C^{1}$ cylinders, Proc. Sympos. Pure Math., vol. 35, part 2, Amer. Math. Soc., Providence, R.I., 1979, pp. 179-196.

[KW] R. Kaufman and J. M. G. Wu, Parabolic measure on domains of class $\mathrm{Lip}_{1 / 2}$, Compositio Math. 65 (1988), 201-207.

[LeS] J. L. Lewis and J. Silver, Parabolic measure and the Dirichlet problem for the heat equation in two dimensions, Indiana Univ. Math. J. 37 (1988), 801-839.

[Mu1] M. A. M. Murray, Commutators with fractional differentiation and BMO Sobolev spaces, Indiana Univ. Math. J. 34 (1985), 205-215.

[Mu2] _ Multilinear singular integrals involving a derivative of fractional order, Studia Math. 87 (1987), 139-165. 
[St] E. M. Stein, Singular integrals and differentiability properties of functions, Princeton Univ. Press, Princeton, N. J., 1970.

[Str] R. S. Strichartz, Bounded mean oscillation and Sobolev spaces, Indiana Univ. Math. J. 29 (1980), 539-558.

[T] A. Torchinsky, Real-variable methods in harmonic analysis, Academic Press, Orlando, 1986.

[Z] A. Zygmund, Trigonometric series, vol. I, Cambridge Univ. Press, Cambridge, 1959.

Department of Mathematics, University of Kentucky, Lexington, Kentucky 40506 0027

School of Mathematics, University of Minnesota, Minneapolis, Minnesota 55455

Current address : Department of Mathematics, Virginia Polytechnic Institute and State University, Blacksburg, Virginia 24061-0123 